\title{
Night Shift Work and Weight Gain among Female Filipino Nurses
}

\author{
Apellido $\mathbf{R}^{*}$
}

Assistant Nurse Manager, New York-Presbyterian/ Queens Hospital, USA

${ }^{*}$ Corresponding author: Apellido R, Assistant Nurse Manager, New York-Presbyterian/ Queens Hospital, USA, Tel: 15162445906, E-mail: armalong56@gmail.com

Citation: Apellido R (2018) Night Shift Work and Weight Gain among Female Filipino Nurses. J Obes Overweig 4(1): 106. doi: 10.15744/2455-7633.4.106

Received Date: February 05, 2018 Accepted Date: May 28, 2018 Published Date: May 30, 2018

\begin{abstract}
There are increasing numbers of overweight and obese nurses working the night shift. The purpose of this correlational and cross-sectional study was to determine if there are statistically significant associations between insufficient sleep, abnormal eating patterns, working 12-hour shifts, years of working night shift, age, stress, marital status, nursing units, decreased physical activity, and level of weight gain among female Filipino nurses working the night shift in the hospital. The theoretical foundation of this study was locate evidence, evaluate evidence, assess evidence, and informed decision model. An online survey through Survey Monkey was used to access nurses via a convenient sampling. Data were analyzed using Spearman correlation, multiple regression, and ANOVA. According to study findings, there were significant associations between insufficient sleep, abnormal eating patterns, marital status, 12-hour shifts, number of years working on night shift, and increased in body mass index. The implications for social change include information that nurses can use to better understand the negative implications of night shift work on health. At the organizational level, this study provides information for administrators and nursing leaders that might facilitate change in policies by improving working conditions for nurses.
\end{abstract}

Keywords: Night Shift; Nurses; Lack of Sleep; Stress; Physical Activity; 12 hours shift

\section{Introduction}

Shift work, particularly night shift work, is an increasing part of the working schedule. Night shifts allows services to be provided around the clock, night shift work helps meet the demands of consumers in many businesses and industries. These businesses and industries include health care, transportation, law enforcement, communication, and manufacturing [1]. Health care providers, such as nurses, provide 24-hour patient care services in hospitals on different shifts. Nurses opt to work night shift for various reasons, including family obligations, babysitting issues, returning to school, and night differential pay. However, despite their work-related knowledge of health promotion, health education, and disease processes [2], nurses are often unaware of the negative health implications of working night shifts [3-6].

Night shift workers experience insufficient sleep, tiredness, and an inability to recover between shifts [7]. Furthermore, Kim, Jeong, and Hong (2015) reported that insufficient sleep, even for one night, could cause energy expenditure and metabolic imbalance that have been associated with weight gain [8]. Additionally, insufficient sleep can increase food consumption [9] by creating a need to replace sleep loss and energy by consuming more food [10].

Scholars have found that night shift nurses are at increased risk of weight gain. Hawkes et al. (2015) observed that attitudes and behaviors concerning food and body weight vary by culture [11]. However, researchers have not focused on the increased risk of weight gain in female Filipino nurses while working the night shift. This study was designed to explore the relationship between night shift work and weight gain among female Filipino nurses.

The implications for positive social change of this study include providing information that might facilitate changes in policies and working conditions that promote the health and wellbeing of nurses. Enhancing the health of the nursing workforce on the night shift and promoting sleep-hygiene initiatives could prevent the development of conditions associated with weight gain, such as heart diseases, hypertension, diabetes, stroke, and some forms of cancer (Center for Disease Control and Prevention [CDC], 2014). Also, according to the CDC (2014), the expected medical costs for overweight people were $\$ 1,429$ higher annually than the costs of those of normal weight [12]. Therefore, enhancing the health of the nursing workforce on the night shift and promoting sleep-hygiene initiatives may have implications for decreased health care expenditures. 


\section{Background of the Study}

Scholars have addressed the factors associated with an increased risk of weight gain among female nurses who work the night shift. Han, Trinkoff, Storr, Geiger-Brown, and Park (2012) and Amani and Gill (2013) reported that stress and lack of sleep during night shifts contributed to nurses' weight gain. Nurses who worked 12-hour night shifts suffered increased fatigue due to insufficient sleep [13-15]. Insufficient sleep, coupled with the abnormal eating habits of night shift nurses, led to weight gain [4]. Nahm, Warren, Zhu, and Brown (2012) discovered that 53.8\% of their participants missed meals from because of busy work schedules. In addition, $72.2 \%$ of the respondents indicated that they felt they were not engaging in enough exercise [16].

Weight gain among night shift nurses is measured by using body mass index (BMI), and being overweight is defined as having a BMI of 25 or greater [12]. Huth, Eliadis, Handwork, Englehart, and Messenger (2012) revealed that 27.5\% of the 97 night shift nurses they studied had a BMI of 30 or above [17]. Huth et al. also found that night shift nurses' quality of sleep was lower than the sleep quality of day shift nurses [17]. Additionally, Marqueze, Soares, Lorenzi-Filho, and Moreno (2012) revealed that working night shifts was associated with greater weight gain than working day shifts and that their participants had significant increases in their BMI compared to day shift nurses [18]. According to Theorell-Haglöw, Berglund, Janson, and Lindberg (2013), both short and long sleeper night shift nurses were centrally obese and had greater neck circumference [19]. Short sleepers were more centrally obese than the long sleepers were.

\section{Problem Statement}

The increasing numbers of overweight and obese nurses working night shifts are becoming the focus of occupational researchers. Researchers have conducted studies on weight gain among nurses from many countries. These countries include South Africa, Australia, the United Kingdom, New Zealand [14,20-22], the Republic of Korea [23] Malaysia [24], the United States [2,15,17], Brazil [18],Taiwan [6], Europe [25] and Canada [26,27] argued that because of the increase of business process outsourcing (BPO) in the Philippines, especially contact and call center work, research on shift work is becoming increasingly important. However, researchers have not focused on weight gain among female Filipino nurses working the night shift. Because norms about food and body weight vary from culture to culture [11], it is important to gain information on this cultural population concerning weight gain among night shift nurses.

Weight gain can lead to obesity. Obesity has become one of the most common health problems worldwide [28]. Having a BMI of 25 or higher constitutes being overweight and having a BMI of 30 or higher constitutes obesity [12,29].The WHO (2015) reported that around 1.9 billion adults 18 years and older were overweight and that 600 million were obese. In the United States alone, 78.6 million are reported as being obese [12]. Because of the prevalence of unhealthy weight gain in night shift nurses, researchers are focusing on the occupational-related weight gain associated with night shift work. For example, Asaoka et al. (2013) found connections between night shift work and weight gain among female night shift nurses in Japan. In Brazil, the problems of night shift nurses were poor sleep quality and reduced access to quality food choices [18]. Working night shifts could also lead to unhealthy weight gain and weight-related medical conditions among female Filipino nurses. However, researchers have yet to focus on female Filipino nurses and night shift work as it relates to weight gain. In this quantitative study, I examined the connections between night shift work and weight gain among female Filipino nurses working in community hospitals in the United States.

\section{Purpose of the Study}

The purpose of this quantitative study was to gather information on whether insufficient sleep, abnormal eating patterns, stress, nursing units, working 12-hour shifts, age, marital status, number of years on night shift, and decreased physical activity are linked to weight gain among female Filipino nurses working the night shift in community hospitals in the United States. Scholars have shown high correlations between night shift work and weight gain [21]. However, little is known about how the factors of night shift work relate to weight gain among female Filipino nurses. Night shift work includes factors such as insufficient sleep, poor sleep quality, poor eating habits, stress, 12-hour shift, and limited physical activity, which may all be related to weight gain among female Filipino night shift nurses [6,21]. Marqueze et al. (2012) suggested that further studies are needed to investigate the relationships between short sleep durations of night shift nurses [18]. Also, further investigation is required on the onset of related metabolic syndromes of weight gain, such as diabetes, hypertension, and high cholesterol levels.

\section{Research Question and Hypotheses}

Research Question 1: What statistically significant associations are there between insufficient sleep, abnormal eating patterns, and level of weight gain among female Filipino nurses working the night shift in the hospital?

$\boldsymbol{H}_{\mathbf{0}} \mathbf{1}$ : There are no statistically significant associations between insufficient sleep, abnormal eating patterns, and level of weight gain among female Filipino nurses working the night shift in the hospital.

$\boldsymbol{H}_{\boldsymbol{a}} \mathbf{1}$ : There are statistically significant associations between insufficient sleep, abnormal eating patterns, and level of weight gain among female Filipino nurses working the night shift in the hospital. 
Research Question 2: What statistically significant associations are there between working 12-hour shifts, number of years working on the night shift, and level of weight gain among female Filipino nurses working the night shift in the hospital?

$H_{0}$ 2: There are no statistically significant associations between working 12-hour shifts, number of years working on the night shift, and level of weight gain among female Filipino nurses working the night shift in the hospital.

$H_{\mathrm{a}}$ 2: There are statistically significant associations between working 12-hour shifts, number of years working on the night shift, and level of weight gain among female Filipino nurses working the night shift in the hospital.

Research Question 3: What statistically significant associations are there between age, marital status, and level of weight gain among female Filipino nurses working the night shift in the hospital?

$\boldsymbol{H}_{\mathbf{0}}$ 3: There are no statistically significant associations between age, marital status, and level of weight gain among female Filipino nurses working the night shift in the hospital.

$H_{\mathrm{a}}$ 3: There are statistically significant associations between age, marital status, and level of weight gain among female Filipino nurses working the night shift in the hospital.

Research Question 4: What statistically significant associations are there between stress, nursing units, decreased physical activity, and level of weight gain among female Filipino nurses working the night shift in the hospital?

$\boldsymbol{H}_{\mathbf{0}}$ 4: There are no statistically significant associations between stress, nursing units, decreased physical activity, and level of weight gain among female Filipino nurses working the night shift in the hospital.

$\boldsymbol{H}_{\mathrm{a}}$ 4: There are statistically significant associations between stress, nursing units, decreased physical activity, and level of weight gain among female Filipino nurses working the night shift in the hospital.

\section{Nature of the Study}

In this study, I employed a quantitative, correlational, cross-sectional design, with data collection conducted via survey (See Appendix B). Correlational researchers assess the linear association between two or more variables in a non-experimental study, and also determine the degree of relations between variables [30]. When using cross-sectional design, the data are collected at a single point in time [31]. Surveys are the most common method of data collection in quantitative research.

The combination of Pearson and Spearman correlations were used to address the research questions. Correlations were used to examine the relationship between the characteristics of female Filipino nurses and weight gain. The significant variables were placed into a multiple linear regression to examine the predictive nature of these characteristics in the prevalence of weight gain.

\section{Assumptions}

It was assumed that female Filipino nurses who work night shift have the same risk of becoming overweight and obese as those of other cultures and races. However, this assumption cannot be demonstrated to be true because no research has been conducted on night shift work and weight gain among this population. In this quantitative study, it was assumed that the participants provided the most accurate information to the questionnaire. After participants were informed of the nature of the study, as well as of the confidentiality of the data collected, it was assumed that the participants answered truthfully and honestly so that the validity of the data is highly regarded for statistical analysis. The BMI of this population was calculated solely on participants' self-reported height and weight. It was assumed that BMI is a valid and accepted measurement for level of weight gain.

\section{Scope and Delimitations}

I focused on female Filipino nurses who were working the night shift in community hospitals in the United States. The demographic characteristics of the participants included age, marital status, education, and the number of years nurses have worked on the night shift. Because of the specifics of culture and ethnicity of female Filipino nurses, the findings of this study cannot be generalizable to other populations in other settings. Further study is needed on this population to compare findings to studies on other populations.

Because the methodology of the study is cross-sectional using questionnaire surveys, causal links between other variables cannot be identified. The purpose of the study was to gather statistical data from female Filipino nurses who were continuously working on the night shift and occupational factors that lead to weight gain. Such factors included poor sleep quality, short sleep duration, stress, limited physical activity, and patterns of eating to compensate lost energy. The exclusions were those who worked less than 3 years on night shifts, work per diem (once a week), familial history overweight, those with chronic medical conditions taking medications (such as steroids), and those in the supervisory or educator roles.

\section{Limitations}

One limitation was that participants' weight and height cannot be objectively measured to calculate their actual BMI measurement; the BMI of this population was based only on participants' self-reported height and weight. Consequently, the effects of social 
desirability bias may come into play. Social desirability bias refers to participants sometimes answering in ways that show them favorably to others rather than answering truthfully [32], especially in studies on topics that will be difficult for individuals to address, such as weight gain. Consequently, self-reported weight gain may be subject to social desirability bias.

Other limitations included self-reported hours of sleep duration; sleep quality, degree of physical activity, and food consumption to replenish their lost energy. Probability sampling was another limitation in quantitative study because this type of sampling does not reflect the larger population. In addition, there were other potential biases in the study. For example, I am a registered nurse who worked on the night shift and possible data manipulation can be a potential risk. In addition, I focused on a cultural population and cultural background can inform attitudes toward diet, consumption, food choices, and physical activity, findings from this study cannot be generalized to other populations.

\section{Significance of the Study}

Unhealthy weight gain is a health issue. This study may help nurses understand that night shift work is associated with many health concerns. These concerns include unhealthy weight gain, cardiovascular diseases, hypertension, premature death, and early disability [12]. Although nurses possess adequate knowledge about health education, health promotion, and nutritional habits, they may not be aware that they are at risk for weight gain [2]. Moreover, night shift nurses may be unaware of the detrimental effects of night shift work on their health [33]. Female Filipino nurses may also unknowingly run the risk of potential unhealthy weight gain. Information collected from this study may help to make nurses aware of the potential health risks associated night shift work.

Information collected from this study also has implications for theory and practice. Information on night shift work and weight gain among female Filipino nurses is lacking in the literature and is needed to identify potential health problems associated with night shift work and weight gain in this population. Information from this study may also have implications for practice and may lead to changes in policies and working conditions to promote the health and wellbeing of the nursing workforce. To achieve this change, nursing leaders and health care administrators need information to initiate health promotion initiatives. These efforts might include better sleep hygiene for nurses to decrease the risk factors associated with working night shifts [34]. Furthermore, information obtained from this study could lead to positive social change. First, the potential positive social change from this study includes providing information that might facilitate changes in policies and working conditions to promote the health and wellbeing of the nursing workforce. Secondly, the information from this study might help nurses better understand that night shift work can be associated with various health problems. Finally, information provided by this study might help to improve the health status of nurses that could decrease medical expenditures to the current health care system.

\section{Literature Search Strategy}

The scholarly literature used for this literature review was accessed mainly through Walden University Library's online databases. The search engines used included the Cumulative Index to Nursing and Allied Health Literature (CINAHL), MEDLINE, PubMed, Health and Allied Complete, Ovid Nursing Journals, and Science Direct. In addition, I used Google Scholar to find current, peer-reviewed literature and searched the WHO and CDC websites for relevant information on weight gain. The literature was accessed electronically in different formats, such as HTML and PDF formats. The key search terms and combinations of terms used included weight gain, nurses, night shift, stress, extended work hours, and elevated BMI. The electronic search of these scholarly journal articles and literature was also customized by publication date. Journal articles were sorted out by the published date from 2012, as the oldest, to 2016, as the most recent studies related to night shift work, nurses, and weight gain. One of the selected works in the literature review is a thesis, and another is a dissertation, which was also a poster presentation at the Council for the Advancement in Nursing Science 2010 in Washington, D. C.

\section{Theoretical Foundation}

There are many theoretical frameworks available for the study of the prevention of adult weight gain; however, the framework most appropriate for this study was a systems-oriented multilevel model developed by Huang et al. (2009) [35]. In 2008, Kaiser Permanente, in partnership with the CDC, asked the IOM committee to develop a framework to address the prevention of weight gain in the public (IOM, 2010). The system-oriented model LEAD (Figure 1) was developed by the IOM to use for a variety of complicated human health issues, including weight gain [36]. According to Huang et al., the model was developed based on the concept of socioeconomic theory, and the model stresses the importance of environmental and social factors related to individual behavior in addressing and controlling for weight gain [35]. Additionally, the LEAD model approaches weight gain through the multifaceted dimensions of individual behavior, and it includes considerations such as food choice, lifestyle, physical activity, cultural influences, and socioeconomic factors [35]. This model has proven successful and effective in addressing other health problems involving the multifaceted dimensions of individual behavior, such as tobacco use and environmental health issues [37].

Unhealthy weight gain has been conceptualized as resulting from a combination of eating too much unhealthy food and low levels of physical activity [35]. However, several factors may also influence the amount and type of food individuals eat, as well as their levels of physical activities [35]. These factors include biology (individual metabolic processes, genetic influences), built 
environment (availability and marketing of foods), social environment (cultural attitudes toward food consumption and body weight), and economics (income and living standards; Huang et al., 2009). At the individual level, weight gain involves attitudes and behavior in personal lifestyle, unhealthy food choices, and lack of motivation [36]. Environmental factors include working conditions, how food is processed and marketed, as well as education about food choices and dietary regimes [29]. Genetic factors include predispositions to dietary illnesses and diseases, as well as familial influences and tendencies [16]. Because of the array of individual, biological, and social influences related to weight gain, scholars have not discovered a definitive way to manage being overweight, but there are preventive programs that can address level of weight gain [36].

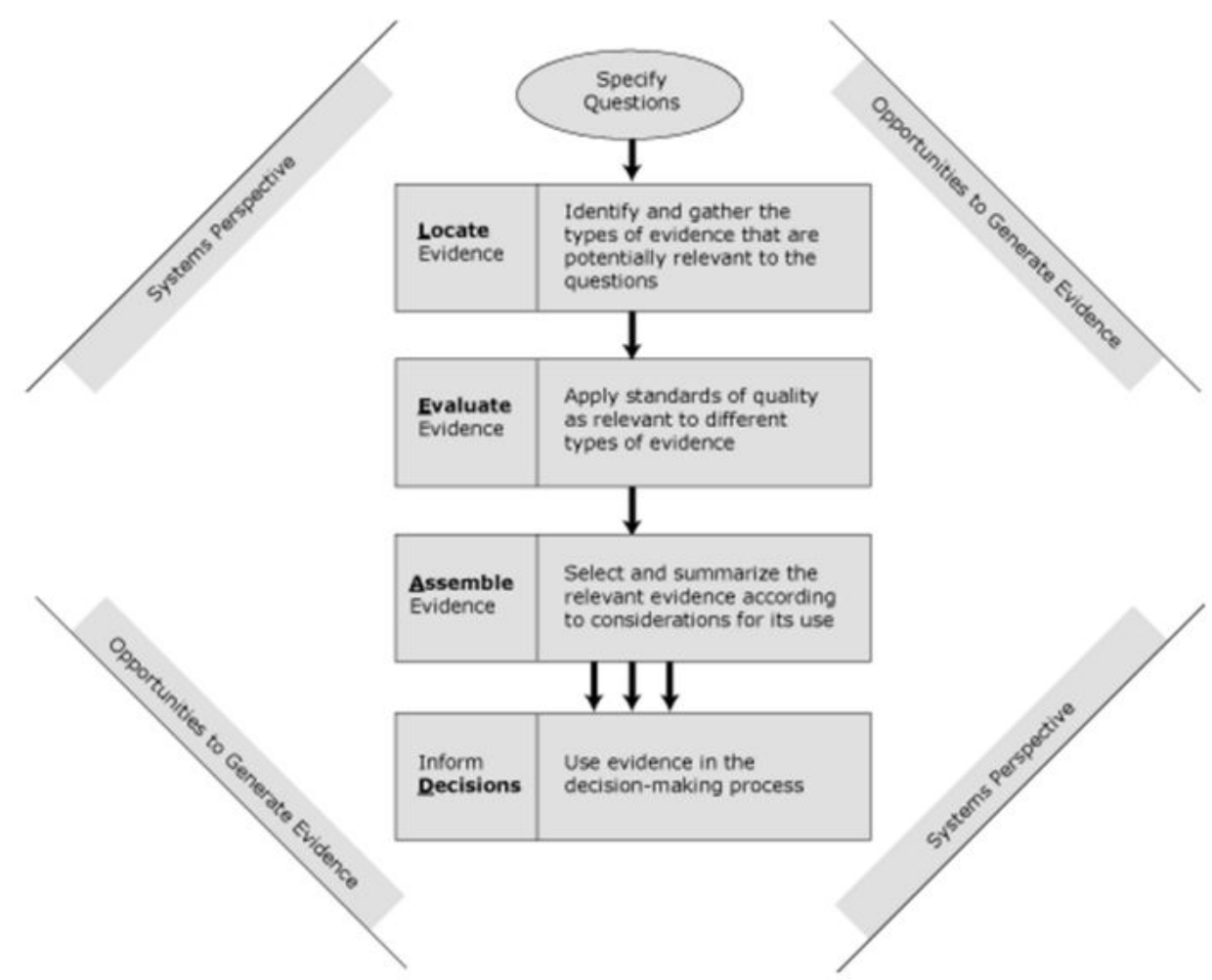

Figure 1: The LEAD framework for weight gain prevention decision making. Adapted from "L.E.A.D.: A Framework for Evidence Gathering and Use for the Prevention of Obesity and Other Complex Public Health Problems," by M. Chatterji, L. W. Green, and S. Kumanyika, 2014, Health Education and Behavior, 41(1), p.87.

Prevention and public health education are the keys to solving the unhealthy weight gain epidemic [16]. Because the causes of unhealthy weight gain are multifactorial, a system-wide approach is needed to study and address this phenomenon. Despite weight gain management used in the past, the rate of unhealthy weight gain continues to increase. The LEAD framework enables policymakers and practitioners to locate the evidence that could advise stakeholders to make decisions regarding the prevention and management of unhealthy weight gain [36]. LEAD provides a practical guide for the implementation of policies in the prevention and management of weight gain in community settings, as well as allowing for the collection of evidence to encourage policymakers and practitioners to understand weight gain as a complex health problem and make informed decisions to curb the unhealthy weight gain epidemic [36].

LEAD includes questions - a what, why, and how guide-in response to locating evidence, evaluating it, assembling it, and informing decision. The why assists decision makers with forming a judgment for taking action on weight gain issues at a geographical location? What includes prevention programs or policies and an evaluation of the effectiveness of the programs or policies being implemented? The how depicts the implementation of the programs that may include resources, support, and the generalizability and transferability of the approach from one setting to the other [37].

The LEAD framework has proved its efficacy in the management and prevention of weight gain through system-oriented decision making and policies. For instance, the prevalence of obesity among children and adolescents aged 2 to 19 years has decreased to $17 \%$ as the result of LEAD-based policies, compared to adult obesity rates of $38 \%$ (CDC, 2014). On policies, schools were encouraged not to serve cold cuts (high in sodium) and calorie-dense snacks. Rather, the school children should increase consumption of fruits and vegetables, replace sweet-sugary beverages with water, and increased physical activity. At the adult level, policymakers educated the public on nutritional facts of the processed food and encouraged the food processing companies to put nutritional labels on their products. Another result of LEAD-based policy is the cessation of the use trans fats in restaurant food preparation that helps in the prevention of weight gain. Chatterji et al. (2014) posited that system-oriented frameworks, like LEAD, are effective because they involve considerations of culture, belief, behavior, and the law in health management [36]. 


\section{Contributory Factors of Weight Gain among Night Shift Nurses}

The causes of unhealthy weight gain in night shift nurses are multifactorial, and individual behavior, environmental factors, socioeconomic status, genetics, and illness play roles in unhealthy weight gain $[18,38,39]$. In this section of the literature review, I focus on the factors associated with unhealthy weight gain among female nurses working the night shift.

\section{Insufficient Sleep}

Sleep is an essential element of good health that helps promote brain functioning $[12,40,41]$ and plays a role in energy metabolism [42]. Insufficient sleep has been associated with long-term medical problems, such as diabetes, heart disease, and depression [12]. In addition, the cumulative effect of insufficient sleep while continuously working night shifts can have negative impacts on nurses' health [5].

Insufficient sleep due to night shift work affects workers' health [43] and the WHO (2015) [29] reported that night shift work could lead to cancer and other health problems. Gu et al. (2015) identified additional problems related to night shift work, including diabetes, high blood pressure, fatigue, sleep problems, and weight gain [43]. Geiger-Brown, Rogers, Trinkoff, Kane, Bausell, and Shcarf (2012) found that insufficient sleep, fatigue, and poor sleep quality attributed to unhealthy weight gain among night nurses [13]. Geiger-Brown et al. reported that nurses usually slept fewer than 6 hours between shifts, meaning that night shift nurses may be sleep deprived [13].

Insufficient sleep promotes hunger and metabolic changes [44]. In addition, Markwald et al. discovered that the association between increased food consumption and insufficient sleep were reactions to the body's need for energy to stay awake at night [42]. Becuti and Pannain (2014) found statistical significance between poor sleep and elevated BMI in women [40]. Becuti and Pannain also observed less sleep and poor sleep quality were linked to the risk of weight gain.

Getting adequate sleep is a component of good health [45]. Healthy People 2020 encouraged adult people to get more than 7 hours of sleep per day that might help promote health and prevent premature deaths [44], and the National Institute of Health encouraged adults to sleep 7 to 8 hours per night [12]. However, Filipino night shift nurses may not be getting the amount of sleep as recommended to ensure good health and prevent weight gain.

\section{Night Shift Work}

Night shift work is becoming more common for workers in various industries, including health care, transportation, manufacturing, and public and consumer services [9,43]. Night shift work refers to working anytime between the hours of 7:00 p. m. and 7:00 a. m, and it has been linked to risk factors leading to weight gain [46]. Because of the impact of night shift work on health, work-related weight gain has been studied [47], and there is a converging body of evidence that night shift work is associated with a variety of health problems, including weight gain [48].

Gu et al. (2015) found that women who worked the night shift for extended durations gained more weight and were more likely to develop diabetes, hypertension, or high cholesterol than day workers [43]. Conversely, Fisharaki et al. (2012) found that night shift workers had lower BMI than day shift workers did; however, these findings do not align with the findings of other studies [46]. Many researchers across the globe are interested in factors connected to work-related weight gain among nurses and in other fields.

Maddox (2013) reported that night shift is a risk factor for weight gain among nurses. Weight gain is one of the main problems related to the health of night shift nurses [17,20] discovered that night shift work increased nurses' risk of having elevated BMI and being overweight compared to the general population. Kim et al. (2013) found that the prevalence of weight gain nurses working the night shift was $5.5 \%$ to $65.4 \%$. Because of negative health consequences associated with night shift work among nurses [49], Asaoka et al. (2013) suggested reducing the amount of night shift work in order to prevent negative impacts on health for night shift nurses [21].

\section{Job Stress and 12-Hour Shifts}

Job stress and long working hours add to night shift nurses' likelihood of weight gain. Han et al. (2012) described nurses' job as stressful. Also, working long hours reduces quality and quantity of sleep and exposes nurses to more stress-promoting food consumption leading to weight gain [15]. Additionally, nurses working 12-hour night shifts slept fewer than 6 hours between shifts, and they complained of fatigue and sleepiness [15]. Night shift nurses may be unaware of the negative impact of lack of sleep on their health and their increased risk of heart problems and other metabolic syndromes, such as weight gain [15].

Kim et al. (2013) revealed that Korean nurses who worked extended period on the night shift have 1.63 (95\% CI, 1.22-2.17) times tendency to be overweight than those who worked short duration [49]. Quist et al. (2013) found that both male and female participants have increased BMI after 3 years [50]. Furthermore, body weight changes occur within the period of 6 months to 1 year, and weight gain was more denoting among participants in the age group of 30 to 40 [51]. Individuals who worked long hours 
on the night shift and slept less were more likely to have an increase in their BMI [52]. Jung and Lee (2015) discovered that night shift causes stress and fatigue on nurses that contributed to their sleep problems [23]. Vijayalaxmi, George, and Nambier (2014) found that night shift workers have higher job stress levels as opposed to the day shift workers [53]. Likewise, stressful working environments, such as those often found in night shift work, put strain on nurses' health [20]. Amani and Gill (2013) reported that long working hours, night shift, and stress have been associated with weight gain and higher BMI in nurses [14].

Compared to other types of work, the work of nurses includes long hours, and nurses are exposed to high level of stress. Boughattas et al. (2014) found that ICU and ER nurses have a heightened stress level compared to nurses in other hospital units [3]. Caruso (2014) found that nurses working 12-hour shifts have a 28\% increased risk of fatigue compared to those working 8- or 10-hour shifts and that working12-hour shifts increased the likelihood of injuries and errors [44]. In addition, Nahm et al. (2012) found that nurses working 12-hour shifts ate their meals at irregular intervals, when compared to nurses working 8-hour shifts, and irregular meals had a negative impact on nurses' health [16].

\section{Age and Duration of Night Work}

As individuals age, the less sleep they need; however, long duration of insufficient sleep does have an impact on health. Kwon et al. (2016) revealed that weight gain was more pronounced among subjects between the ages of 30- to 40-years-old [51]. Kim et al. (2013) found that the longer nurses worked the night shift, the greater their chances of becoming overweight and obese [8]. Quist et al. (2013) found that both male and female Danish health care workers had increased BMI. In similar studies, extended duration of night shift work found to be connected to weight gain, diabetes, and high blood pressure [34,43].

Kim et al. (2013) found that nurses who worked long-term night shifts were 1.63 times gained weight that those who worked short duration [8]. Because of the body's tendency to adapt to a particular work schedule, nurses who work longer than four to five years of night shift are more at risk of developing metabolic syndromes [54], including Type 2 diabetes, hypertension, heart diseases, stroke, and some forms of cancer [12].

People's metabolisms tend to slow down and they tend to become heavier as they age. Letvak, Ruhm, and Gupta (2013) discovered that weight gain increased with age for nurses aged 50 and older; they were also found to have higher BMI than younger nurses [54]. Also, Vimalananda et al. (2015) found that women below 50-years-old who worked night shift for ten years had a 39 percent chance of having diabetes than those who never worked the night shift [55]. Boughattas et al. (2014) reported that nurses' ages influenced the quality and duration of sleep, and Marqueze et al. (2012) found that nurses' ages and night shift work does contribute to weight gain $[3,18]$.

\section{Marital Status}

The marriage market suggests that a married person tends to gain weight because he or she is no longer interested in attracting a mate [56]. Moreover, married people have an increased BMI as a result of marriage pressure [56].

\section{Eating Habits}

Night shift nurses must often sleep during the daytime and, consequently, frequently miss their meals or eat them at irregular intervals. Griep et al. (2014) found in their study of 2,372 nurses that mostly women had elevated BMI due to irregular meals after working the night shift for extended periods of time [38]. Sleep deprived nurses also tend to consume meals high calories and fats to help combat sleepiness [22,38]. In addition, night shift nurse are often unable to maintain regular eating patterns [14]. Moreover, a continued alteration in eating patterns contributed to weight gain [10] that consequently may lead to obesity [21]. According to Depner et al. (2014), epidemiological studies have consistently showed that night shift work is linked to weight gain because insufficient sleep promotes food consumption. Conversely, Nagashima et al. (2014) argued that the linkage of irregular meals to weight gain has not been established [9,22]. Also, Gallant, Lundgreen, and Draper (2012) argued that weight gain is not often related to night eating and sleep-related problems [57]. Meanwhile, Phiri et al. (2014) found that poor dietary habits coupled with physical inactivity were the risk factors for weight gain among night shift nurses [20]. The accumulation of extra body weight over time consequently can lead to unhealthy weight gain not only among night shift workers, but the general population. The researchers' findings corroborated that unhealthy weight gain results from imbalances between food intake and inactivity for an extended period of time, and that the development of unhealthy weight gain is a long-term process that involves food choices and dietary habits.

\section{Lack of Physical Activity}

In comparison to nurses' night shift work, day shift work has more patient activity than night shift work. In contrast to the day shift, the night shift requires less physical exercise because patient is sleeping, but working the night shift is difficult because nurses need to stay awake. After night shift work, nurses are tired, sleepy, and have no energy to engage in any form of exercise. According to Kim et al. (2015), night shift nurses consumed more food to stay awake along with limited activities influenced weight gain [8]. It has been emphasized that a poor dietary choices and less physical movements are the causes of gaining weight [39]. However, because fatigue is one of the major problems of night shift nurses [17], night shift nurses unable to maintain regular exercise are at 
risk of becoming overweight [14]. In addition, nurses who are overtired from a stressful and long night's work are disinclined to exercise [20]. The CDC (2014) reported that physical activity improves health, but adults who do not engage in physical activity have a higher risk of health problems, including premature death, heart disease, stroke, Type-2 diabetes, depression, and some forms of cancer [12].

\section{Obesity Trends in the United States}

Preventing unhealthy weight gain is crucial because unhealthy weight gain can lead to obesity. Obesity ranked 5th in mortality worldwide with estimated deaths of 2.8 million per year [58]. In the United States, the numbers of obese adults were 78.6 million or 34.9 percent [12]. Adult obesity in the United States for middle-aged individuals between the ages of 40 to 59 was 39.5 percent, for young adults age 20 to 39 was 30.3 percent, and for those above 60 was 35.4 percent [12]. In comparison to the 50 states including the District of Columbia, no single state has less than 30 percent of individuals being overweight and less than 21 percent being obese [12]. Alaska has the highest rate of overweight individuals represented by 37.7 percent, while Mississippi and West Virginia have the same rate of obesity represented by 35.1 percent [12].

\section{Obesity among Nurses}

Obesity is the cumulative effect of energy imbalance, such as excessive food intake and decreased energy expenditures [21,24]. It has been documented that obesity among nurses in different cultures and races is increasing, and obesity is becoming a public health issue. Researchers worldwide are compelled to focus their attention on work-related weight gain and obesity among nurses, particularly working the night shift. The impact of weight gain and obesity exacerbate health care costs across the globe [12,29].

Geiger-Brown et al. (2012) found almost 50 percent of the 80 female nurses in a critical care unit were overweight or obese [13]. Findings of other studies indicated that 50.6 percent of 1,086 Malaysian nurses were overweight or obese, and the likelihood of Nigerian nurses being overweight or obese was 62.6 percent [24].These research findings have shown that weight gain and obesity among nurses are prevalent worldwide. In the United States, Huth et al. (2012) conducted a descriptive quantitative, correlational study of 753 pediatric nurses and found that 97 of the 420 nurses had a 30 or greater BMI, and 25.8 had a BMI greater than 30 [17]. In a cross-sectional study, Giurgiu and Bordac (2013) showed 72.8 percent of men were overweight and 25 percent were obese, while 55.8 of women were overweight and 24.4 percent were obese [58].

In other industries, Jermendy, Nadas, Hegyi, Vasas, and Hidvegi (2012) found in their cross-sectional study of factory workers that female night shift workers had higher BMI than day workers did [59]. Saksvik-Louilhier et al. (2012) found in their study that night shift workers had higher BMI compared to day shift workers. Conversely, most studies showed that night shift had higher BMI than day workers did. In their longitudinal study of steel factory workers, Fisharaki et al. (2012) found that night shift workers had lower BMI than day shift worker did [46]. Many study results in other industries are conflicting regarding higher and lower BMI levels between night shift and day shift workers. According to Kim et al. (2013), the result of their cross-sectional study showed that nurses' BMI increased with extended exposure to night shift work [8].

The estimated weight gain of night shift nurses in the study is approximately seven to 10 pounds for nurses who worked 10 years or below, and 11 to 15 pounds for nurses who worked more than 10 years. Marqueze et al. (2012) reported in their study that 52.7 percent of participants had increased BMIs, with a 5.8 kilograms average increase among night shift workers [18]. In addition, Griep et al. (2014) disclosed that for women who worked the night shift for 20 years, the average BMI was $25.6 \mathrm{~kg} / \mathrm{m}^{2}$, and after 20 years it was $26.9 \mathrm{~kg} / \mathrm{m}^{2}$ [38]. A healthy BMI for women is 18.5 to 24.9, and 25.05 to 29.9 indicates being overweight [12]. Moreover, according to Quist, Christensen, Christensen, Borg, and Bjorn (2013), after a three-year follow-up with study participants, men gained $0.25 \mathrm{~kg} / \mathrm{m}^{2}$ and women gained $0.48 \mathrm{~kg} / \mathrm{m}^{2}$ [50]. The conflicting reports of different studies warrant further investigation into whether night shift work is connected to higher BMIs in Filipino night shift nurses.

\section{Obesity Management and Prevention}

Most studies on weight gain and obesity documented that the society has been trying to solve this chronic problem for almost 30 years. A decade ago, obesity rates increased and reached epidemic proportions [12]. Today, there are plenty of infomercials trying to engage the public in any forms of physical activity to control weight gain and obesity. There are also different dietary managements on the market, and some pharmaceutical companies continue to discover drugs to help shed excess body weight. Some of the drugs available are FDA approved and some are not. Despite of all these available forms of weight management on the market, maintaining a healthy body weight can be a challenge [12].

Researchers have suggested ways to help improve the health of night shift nurses. According to Caruso (2014), Healthy People 2020 encouraged adults to get more than seven hours of sleep per day to promote health and prevent premature death from obesity [44]. Markwald et al. (2013) offered public health initiatives including sleep education to avoid weight gain. Nurses need to engage in healthier behavior in order to improve their sleep quality [13]. According to Smith et al. (2012), one way to combat obesity in the workplace is through health promotion programs [26]. Health promotion programs could benefit the organizations through decreased employee absenteeism and reduced medical expenditures. In addition, nutrition program policies must be imposed in 
the community also at the local, state, and national levels. Imes and Burke (2014) advocated that policies must continue to focus on nutrition, dietary education, and promotion of physical mobility to reduce the prevalence of obesity in the United States and across the globe [60].

Because of the negative impact of night shift work on health, many scholars who studied this phenomenon found some solutions to improve the health of individuals who continue to work the night shift. Asaoka et al. (2013) suggested reducing the amount of night shift work for nurses [21]. Another important step in obesity prevention is public education regarding the importance of sleep in the promotion of health. McNeil et al., (2013), and Perry et al., (2013) recommended that adequate night sleep as a critical component of good health $[41,45]$. Nurses need to recuperate and need to gain back the lost sleep, in quantity and quality, when working 12-hour-night shifts. Geiger-Brown et al. (2012) advocated that nurses maintain healthy work schedule and practice sleep hygiene by taking frequent breaks to overcome tiredness [13].

Obesity prevention at the organizational level is another initiative. Phiri et al., (2014) recommended a workplace program to support nurses in dealing with stress and improve working environments in order to facilitate the healthy lifestyle of nurses [20]. This can be achieved by providing healthier food choices in hospital cafeterias. To support these suggestions, organizational policy and program planning should be a priority to improve the health of the nursing workforce [24]. Furthermore, nursing leaders, managers, and healthcare administrators must employ policies in the workplace allowing frequent breaks to reduce the risk of fatigue [44]. In the event that night shift workers have chronic sleep problems that truly impact their health, the use of prescription medication might be necessary to help them sleep. Smith and Eastman (2012) suggested that the best way to fight fatigue and sleepiness while working the night shift was to maintain circadian rhythm through the use of melatonin, hypnotics, and sedatives [48].

\section{Design and Methodology}

\section{Variables}

The independent variables of this study corresponded to physical and biological characteristics of female Filipino nurses. The independent variables included insufficient sleep, abnormal eating patterns, stress, nursing units, work 12-hour shifts, age, marital status, number of years on night shift, and less physical activity. Quist et al. (2013) found increased BMI among male and female study subjects [50]. According to Kwon et al. (2016), night shift induced weight change among female participants between the aged of 30 to 40 [51,61]. The dependent variable for this study corresponded to level of weight gain, as measured by BMI $[12,29,62]$. The demographic characteristics included age, gender, marital status, and education.

\section{Research Design}

Research designs are procedures and plans for studies that include the methods of data collection and analyses [63]. I used a quantitative methodology, with a correlational, cross-sectional, survey approach. Quantitative methodologies are systematic investigations of a problem that can be measured using numerical or statistical data [64]. The key dependent variable for this research was BMI, which is a numerical measurement. Correlational researchers assess the linear association between two or more variables in non-experimental studies and determine the degree of relations between two variables [30]. Cross-sectional approaches are used when the data are collected at one point in time that often involves questionnaire survey [31]. An experimental or quasiexperimental approach would not be suitable for this research because observations were not randomly assigned to treatment and control groups, and there was not an intervention being conducted. An online survey approach is appropriate when the variable measurements of interest obtained through participants' self-reported responses are fast and cost effective for data processing [65]. The responses obtained from the survey were helpful in describing the statistics and trends in Filipino nurse weight gain.

\section{Population}

The population of this study was female Filipino nurses who were working during the night shift as a registered nurse in community hospitals in the United States. The characteristics of the target population included women working 75.0 hours per pay period (2 weeks) for full time and or 37.5 hours per pay period for part time, working 3 or more years as a nurse, aged 30- to 62-years-old, and Philippines-educated nurses who met the requirements to practice in the United States.

\section{Sample Procedure}

The sample for the study consisted of a group of female Filipino nurses working the night shift. Convenience sampling was the type of sampling used for the recruiting process. Convenience sampling refers to the selection of participants due to their proximity and accessibility to the researcher [66]. The sample used in this study was based on the data collected from female Filipino nurses. The inclusion criteria for the study were female Filipino nurses who were working the night shift as a registered nurse. Participants were excluded based on the following criteria: male Filipino nurses working as registered nurses, those who were younger than 30-years-old or older than 62-years-old, had gestational weight gain before starting work as a registered nurse, or were overweight prior to working as a nurse. Additionally, those who worked fewer than 3 years on night shift, worked per diem (once a week), had familial history of overweight, those with chronic medical conditions taking medications (such as steroids), and those in the 
supervisory or educator roles were also excluded.

\section{Power Analysis}

When conducting research involving statistical analyses, it is necessary to recruit a sufficient sample size to validate the statistical findings. I used correlations with multiple linear regressions. A conventional power of .80 and an alpha level of .05 will ensure a 95\% statistical certainty that the findings did not occur by chance alone [67].

For the multiple linear regression, a medium effect size was used, $f^{2}=.15$. A total of nine predictor variables were entered into the model. Applying the above parameters to $\mathrm{G}^{\star}$ Power 3.1.7 statistical software, it was determined that a sample size of 98 participants would be sufficient for the multiple linear regression [68]. Assuming a 20\% return rate on the surveys, I invited 500 nurses for participation in the study. In the event that I was unable to meet the minimum requirements of participants, I would reach out to another institution and extend the data collection process. Hardigan, Succer, and Fleisher (2012) suggested that if no response is received after 2 weeks, a follow-up e-mail is necessary and, after 1 week, a final reminder should be sent to yield $99 \%$ responses on a web-based survey [69]. According to Keusch (2012), multiple contact strategies to participants' increases response rates, such as follow-up calls, sending a short message reminder, and text messages [70].

\section{Procedure for Recruitment, Participation, and Data Collection}

\section{Recruitment and Participation}

I recruited participants through an invitation letter sent through e-mails. Participants' names, home addresses, and e-mails were obtained through the hospital's human resources record (see Appendix A). The invitation letter was e-mailed to the participants and explained the purpose and goals of the research. The survey was conducted in New York-Presbyterian/Queens Hospital in Flushing, New York. The survey questionnaire was linked to Survey Monkey, and the instructions to the link were provided. Participants were advised to answer the survey questionnaire and provide the best possible or honest answers to the questions. Participants were informed that they can stop or may refuse to answer any questions at any time. I recruited participants through an invitation letter sent through e-mails. Participants' names, home addresses, and e-mails were obtained through the hospital's human resources record (see Appendix A). The invitation letter was e-mailed to the participants and explained the purpose and goals of the research. The survey was conducted in New York-Presbyterian/Queens Hospital in Flushing, New York. The survey questionnaire was linked to Survey Monkey, and the instructions to the link were provided. Participants were advised to answer the survey questionnaire and provide the best possible or honest answers to the questions. Participants were informed that they can stop or may refuse to answer any questions at any time.

\section{Data Collection}

The data collection process represents the recipe format for sampling and administering surveys to participants [63]. Data were collected from a researcher-created survey tool based on literature review that was administered to prospective participants. Prior to completing the survey, the participants were required to provide consent for their involvement. In the survey, I asked participants to indicate their levels of sleep, stress, work hours, and eating habits. The Likert-scaled responses to these items were used as the independent variables or predictors in the study. In the survey, I also recorded participants' height and weight, which were used for the calculation of BMI. BMI was treated as the dependent and criterion variable for the research questions. Two previously validated survey instruments were used in the study: the Pittsburg Sleep Quality Index (PSQI) and the Perceived Stress Scale (PSS-14). The data used in this study were in compliance with the requirements for the use of human subjects of the Institutional Review Board (IRB) of Walden University. The IRB approval number was 03-06-17-0173570, and it was obtained prior to any data collection.

\section{Pittsburg Sleep Quality Index}

The PSQI contains 19 self-rated questions regarding sleep quality. Sample questions include "During the past month, when have you usually gone to bed at night?" and "During the past month, when have you usually gotten up in the morning? A combination of free response and multiple choice items were used. The PSQI generates seven scores that correspond to subjective sleep quality, sleep latency, sleep duration, habitual sleep efficiency, sleep disturbances, use of sleep medications, and daytime dysfunction. Combining the factors together generates a global score, ranging from 0-21. A PSQI global score greater than 5 is typically considered to be evidence for significant sleep disturbance. The PSQI has previously demonstrated acceptable reliability for the overall scale, $\alpha=.83[71]$.

\section{Perceived Stress Scale}

The PSS-14 contains 14 self-rated survey items regarding stress (see Appendix C). Sample items include "In the last month, how often have you been upset because of something that happened unexpectedly?" and "In the last month, how often have you felt nervous and "stressed? "The PSS-14 scores were obtained by a sum of all 14 items. Seven items were reverse scored prior to calculating the composite scores. Total scores can range between 0-56. The PSS-14 has previously shown an acceptable level of reliability, $\alpha=.78[61]$. 


\section{Dependent Variable}

Level of weight gain was the primary dependent variable of the study. Being overweight is classified as having a BMI of 25 or greater $[12,29,62]$. BMI was calculated based on the participants' self-reported height and weight. Participants were asked "How tall are you without shoes?" [12]. Responses were provided in feet and inches. Participants were also asked, "How much do you weigh without shoes?" Responses were recorded in pounds.

\section{Data Analysis Plan}

Raw data from the survey responses were entered into SPSS version 24.0 for Windows. Frequencies and percentages were examined for the nominal variables of interest such as gender. Descriptive statistics was used to examine the means and standard deviations of continuous variables. Bar charts and histograms were used to visually examine the trends in the data. Data were examined for outliers by calculation of standardized values, or $z$-scores.

To address the research questions, a combination of Pearson and Spearman correlations were conducted to examine the associations between the characteristics of female Filipino nurses and level of weight gain. Pearson correlations are appropriate statistical analyses when the goal of the research is to measure linear relationship between two variables [72]. Spearman correlations are used to assess the strength of association between two variables when at least one of the variables is measured on an ordinal scale [72]. Prior to analysis, the assumption of linearity was assessed. Each correlation will have a coefficient value and an associated $p$-value. $P$-values less than .05 will indicate the presence of a significant relationship. Cohen's standard will be used when interpreting the strength of correlation coefficients. Correlation coefficients between .10 and .29 represent a small association; values between .30 and .49 represent a medium association; and values above .50 represent a large association [73].

The significant variables in the correlations were entered into a multiple linear regression analysis. A multiple linear regression is an appropriate statistical analysis when the goal of the research is to assess the predictive relationship between a set of predictors and a continuous criterion variable [74]. The criterion variable corresponded to BMI. Prior to conducting the multiple linearregression, the assumptions of absence of multicollinearity, normality, and homoscedasticity will be assessed by examination of scatterplots.

The multiple linear-regression used the $F$ test to evaluate the collective effect of the predictors on the criterion variable. If the overall regression model is significant, individual $t$ tests will be conducted on each predictor value to assess the strength of each predictor. $P$-values less than .05 will suggest the presence of a significant relationship and the null hypothesis will be rejected in favor of the alternative hypothesis.

\section{Threats to Validity}

Threats to external validity correspond to areas of the sample that provide bias in regards to the interpretation of the findings [75]. I used sample from Filipino nurses in the hope that the findings of the statistical analyses can be linked to the greater population. However, extreme caution was used in the interpretation of these findings and it will not be assumed that the statistical findings can be perfectly generalized to the population of interest.

Threats to internal validity correspond to potential limitations existing in the situational specifics of the research design and data collection steps. First, there may be several confounding variables that explain the relationship between the variables of interest. The researcher acknowledged the potential effect that these confounding variables are having on the statistical findings. In addition, the selection of a quantitative methodology has potential limitations. While quantitative methodologies can statistically examine research questions, these studies are unable to accurately assess the underlying experiences and perceptions of subjects.

\section{Ethical Considerations}

Researchers have an ethical responsibility to inform and protect all participants involved in the study. Any possible ethical conflicts were avoided by carefully planning all components of the study. I followed the moral and ethical guidelines identified by federal regulations and the Institutional Review Board (IRB) of Walden University. Prior to administering surveys to the participants, the IRB approved all steps of data collection. The identity of participants was protected and their anonymity remained confidential. All information inputted to SPSS was handled personally and was protected through encrypted password in my laptop computer. The safeguard measure for data storage is a locked file within my residence, where the data will be securely maintained for a period of five years after the research is completed. Following the expiration of the five-year retention period, all research-related data will be permanently deleted.

\section{Data Collection}

The study was both approved by the IRB of Walden University and the New York-Presbyterian Hospital/Queens Hospital. Data were collected through the Internet via Survey Monkey ${ }^{\mathrm{TM}}$ from March 27, 2017 to April 15, 2017. Initially, there were 115 female Filipino registered nurses recruited to participate in the study who were conveniently selected from the records in the nursing 
office of New York-Presbyterian/Queens Hospital. Upon evaluation for inclusion in the study, 17 registered nurses were excluded, two were on medical leave, four were on orientation, and 11 were employed less than 3 years and they were younger than 30 -yearsold. Invitations were sent to 98 eligible participants through their respective hospital e-mails on March 20, 2017. Data were collected from 75 participants' responses. Prior to analysis, the data were screened for missing cases and outliers. There were a total of seven removals made due to participants not answering completely to a majority of the questionnaire's items. Outliers were examined by calculation of standardized values, or $z$-scores. $Z$-scores falling outside the range \pm 3.29 standard deviations away from the mean were considered outliers. One case was subsequently removed for an outlying response. After reductions, the data were analyzed from the complete responses of 67 participants.

\section{Demographic Characteristics of Participants}

Descriptive statistics for demographic data were collected, such as age, marital status, and level of education. Age was subgrouped into 30 to 34, 35 to 40, 41 to 45, 46 to 50, 51 to 55, and 55 and over. Marital status was described as single, married, divorced, separated, and widower. Educational levels were subcategorized into associate degree or diploma in nursing, BSN, MSN/MS, and $\mathrm{PhD} / \mathrm{DNP}$ or other doctoral level. The ethnicity was Asian.

\section{Results}

\section{Frequencies and Percentages}

Frequencies and percentages were examined for the nominal and ordinal level variables. Age 30- to 34-years-old represented the most among the participants $(n-14,21 \%)$ followed by age 46 - to 50 -years-old $(n=13,19 \%)$. A majority of participants were married $(n=50,75 \%)$. A majority of the samples were employed full time $(n=61,91 \%)$ and had a BSN $(n=55,82 \%)$. Most of the participants worked night shift $(n=63,94 \%)$ and had shifts extending 12 hours $(n=62,93 \%)$. Most of the participants worked in the critical care units $(n=24,36 \%)$ or medical surgical units $(n=29,43 \%)$. A majority of the participants previously worked the night shift before transferring to the day shift $(n=45,67 \%)$. Frequencies and percentages are presented in Table 1 .

\begin{tabular}{|c|c|c|}
\hline Variable & $n$ & $\%$ \\
\hline \multicolumn{3}{|l|}{ Age } \\
\hline 30 to 34 years old & 14 & 21 \\
\hline 35 to 40 years old & 9 & 13 \\
\hline 41 to 45 years old & 10 & 15 \\
\hline 46 to 50 years old & 13 & 19 \\
\hline 51 to 55 years old & 9 & 13 \\
\hline Over 55 years old & 12 & 18 \\
\hline \multicolumn{3}{|l|}{ Marital Status } \\
\hline Divorced & 4 & 6 \\
\hline Married & 50 & 75 \\
\hline Single & 11 & 16 \\
\hline Widow & 2 & 3 \\
\hline \multicolumn{3}{|l|}{ Employment Status } \\
\hline Full time (75.0 hours per 2 weeks pay period) & 61 & 91 \\
\hline Part time ( 37.5 hours per 2 weeks pay period) & 6 & 9 \\
\hline \multicolumn{3}{|l|}{ Nursing Education } \\
\hline Associate Degree in Nursing or Diploma in Nursing & 4 & 6 \\
\hline BSN & 55 & 82 \\
\hline MSN/MS & 8 & 12 \\
\hline \multicolumn{3}{|l|}{ Work Night Shift } \\
\hline No & 4 & 6 \\
\hline Yes & 63 & 94 \\
\hline \multicolumn{3}{|l|}{ Shift Extend to 12 Hours } \\
\hline No & 5 & 7 \\
\hline Yes & 62 & 93 \\
\hline \multicolumn{3}{|l|}{ Specialty Unit } \\
\hline Critical Care Units (ICU, CCU, ER, CVRU) & 24 & 36 \\
\hline Labor and Delivery Unit (Mother/Baby) & 4 & 6 \\
\hline
\end{tabular}




\begin{tabular}{|c|c|c|}
\hline Variable & $n$ & $\%$ \\
\hline Medical-Surgical Unit & 29 & 43 \\
\hline Operating Room/Recovery Room/Cardiac Catheterization Laboratory & 2 & 3 \\
\hline Telemetry Unit & 8 & 12 \\
\hline \multicolumn{3}{|l|}{ Worked Night Before Changing into Day Shift } \\
\hline Never & 4 & 6 \\
\hline No & 18 & 27 \\
\hline Yes & 45 & 67 \\
\hline \multicolumn{3}{|l|}{ Length of Working Current Shift } \\
\hline 3 to 5 years & 20 & 30 \\
\hline 6 to 10 years & 9 & 13 \\
\hline 11 to 15 years & 11 & 16 \\
\hline 16 to 20 years & 11 & 16 \\
\hline 21 to 35 years & 16 & 24 \\
\hline \multicolumn{3}{|l|}{ Hours of Sleep } \\
\hline 3 hours or less & 4 & 6 \\
\hline 4 hours & 20 & 30 \\
\hline 5 hours & 28 & 42 \\
\hline 6 hours & 11 & 16 \\
\hline 7 hours and above & 4 & 6 \\
\hline \multicolumn{3}{|l|}{ Sleep Quality } \\
\hline Poor & 15 & 22 \\
\hline Fair & 33 & 49 \\
\hline Good & 18 & 27 \\
\hline Very good & 1 & 1 \\
\hline \multicolumn{3}{|l|}{ Miss Meal } \\
\hline Breakfast & 11 & 16 \\
\hline Dinner & 33 & 49 \\
\hline Lunch & 16 & 24 \\
\hline Snack & 7 & 10 \\
\hline \multicolumn{3}{|l|}{ Miss Meal } \\
\hline Always/all the time & 10 & 15 \\
\hline Most of the time & 25 & 37 \\
\hline Sometimes & 30 & 45 \\
\hline Not at all & 2 & 3 \\
\hline \multicolumn{3}{|l|}{ Physical Activity } \\
\hline High & 1 & 1 \\
\hline Low & 23 & 34 \\
\hline Moderate & 43 & 64 \\
\hline \multicolumn{3}{|l|}{ Exercise After Work } \\
\hline Never & 12 & 18 \\
\hline Once a week & 2 & 3 \\
\hline Sometimes & 39 & 58 \\
\hline Twice a week & 10 & 15 \\
\hline Thrice a week & 4 & 6 \\
\hline
\end{tabular}

Table 1: Frequency Table for Nominal Variables

Descriptive statistics were examined for the continuous level variables. Global PSQI scores ranged from 1.00 to 12.00 with $M=$ 4.42 and $S D=2.22$. As evidenced by Figure 2, Global PSQI scores were slightly skewed to the right. Perceived stress scores ranged from 8.00 to 33.00 with $M=23.07$ and $S D=5.51$. As evidenced by Figure 3, perceived stress scores were slightly skewed to the left. $\mathrm{BMI}$ values ranged from 18.20 to 32.89 with $M=25.18$ and $S D=2.91$. As evidenced by Figure 4 , BMI values were approximately normal in distribution. Table 2 presents the findings of the descriptive statistics. 


\begin{tabular}{|c|c|c|c|c|}
\hline Continuous Variables & Min. & Max. & M & SD \\
\hline Global PSQI & 1.00 & 12.00 & 4.42 & 2.22 \\
\hline Perceived Stress Scale & 8.00 & 33.00 & 23.07 & 5.51 \\
\hline BMI & 18.20 & 32.89 & 25.18 & 2.91 \\
\hline
\end{tabular}

Table 2: Descriptive Statistics of Continuous Variables

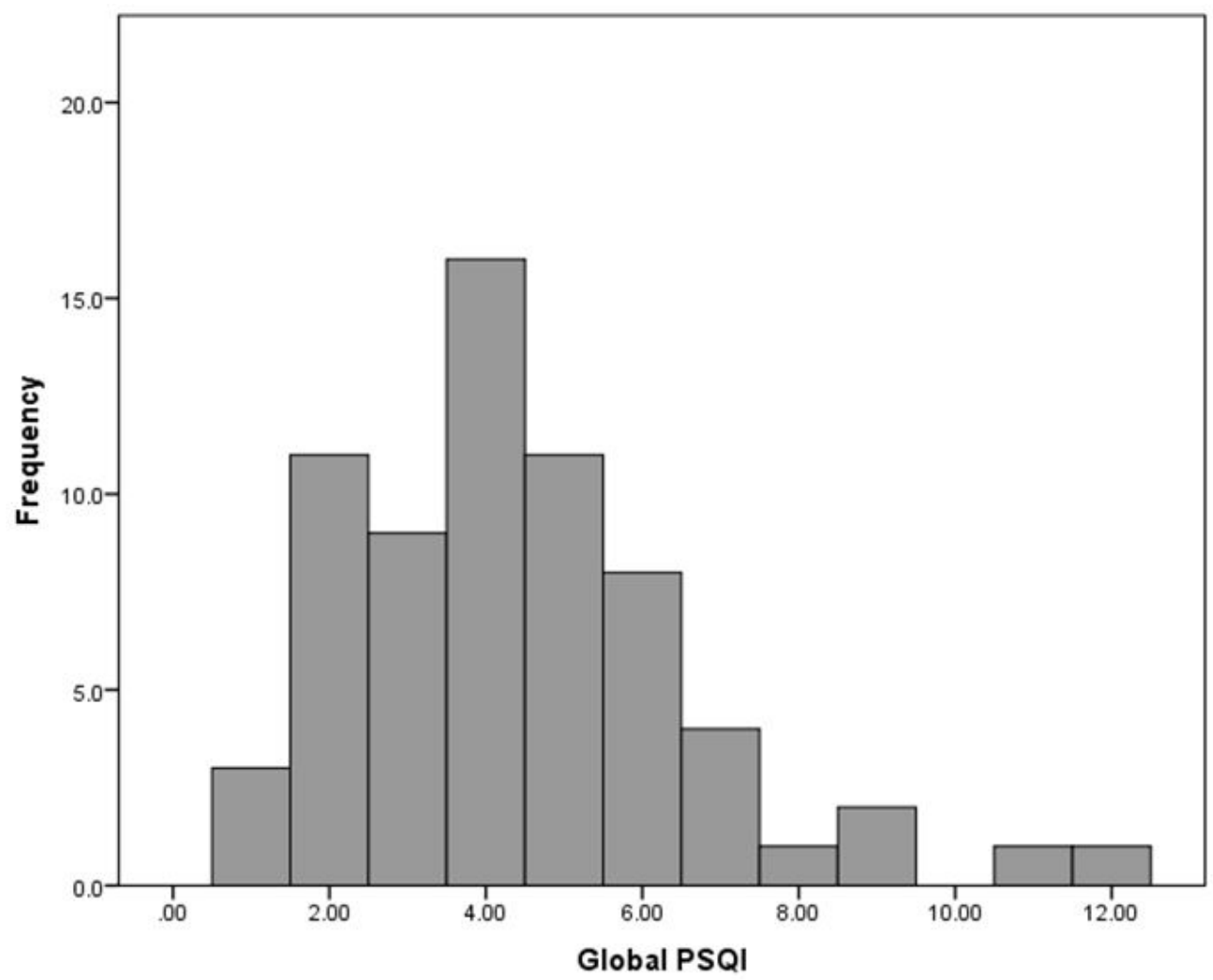

Figure 2: Distribution of global PSQI scores

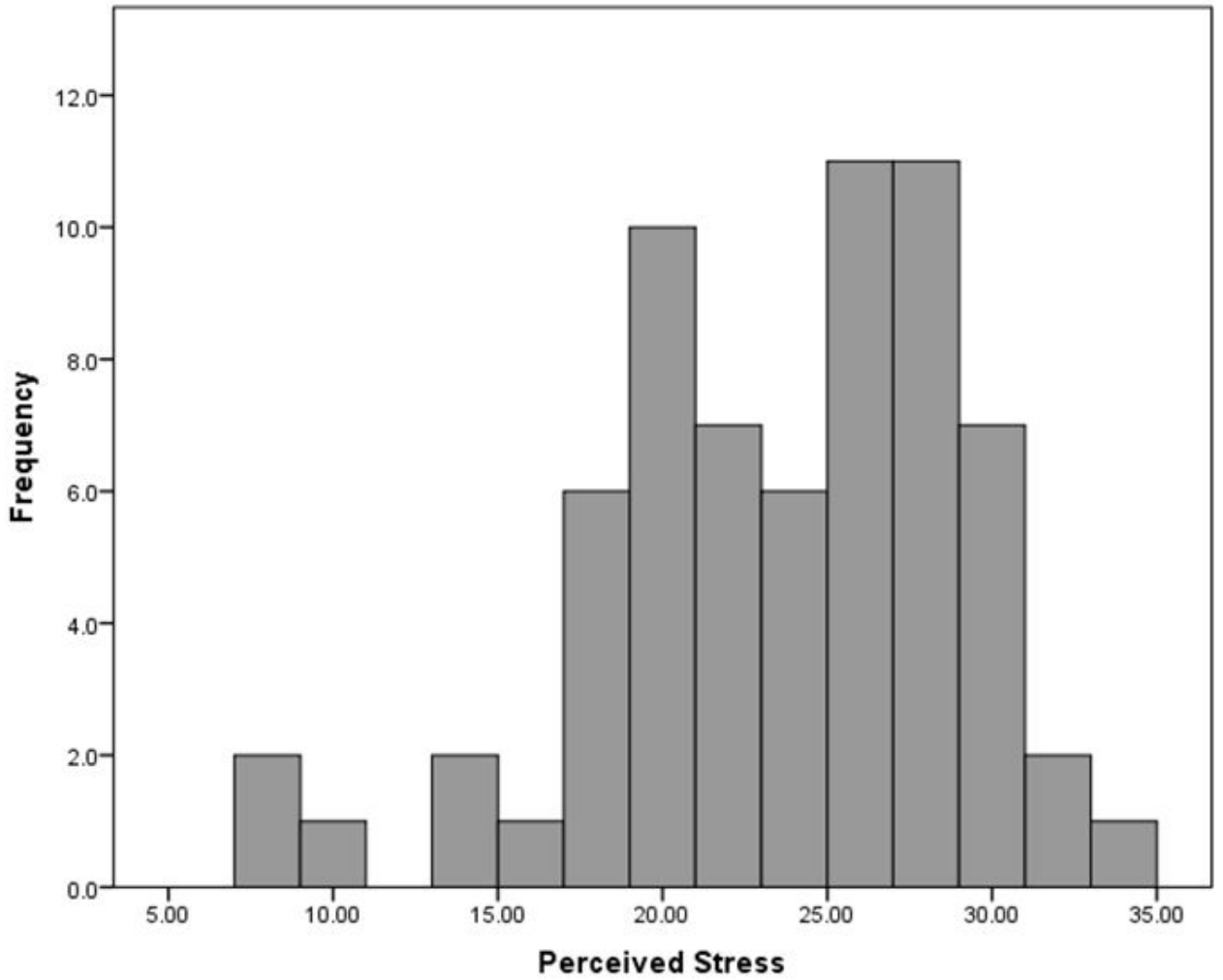

Figure 3: Distribution of perceived stress scores 


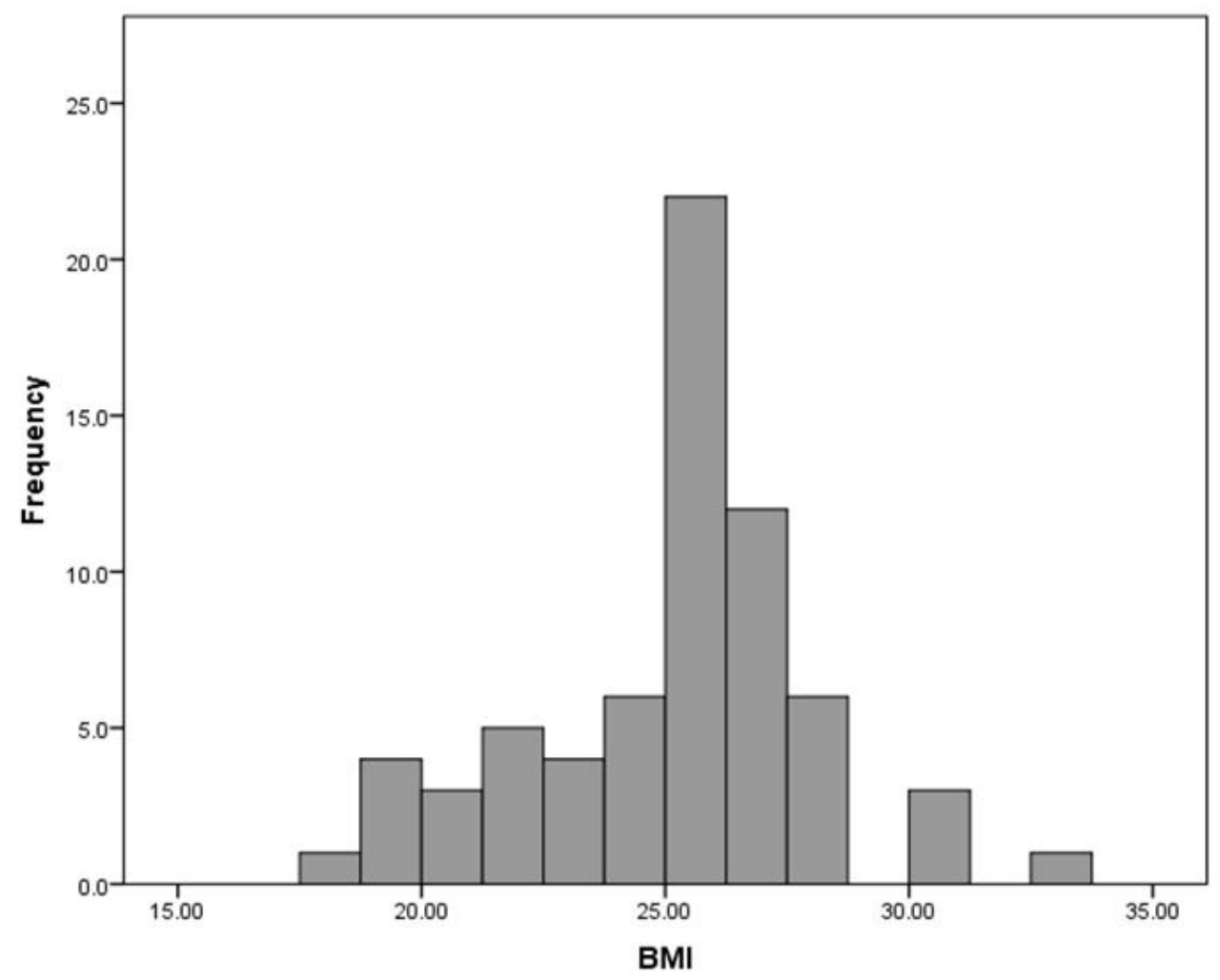

Figure 4: Distribution of BMI values

\section{Detailed Analysis}

To address Research Question 1, a series of Spearman correlations were conducted to examine the two-way associations between average hours of sleep between shifts, sleep quality, frequency of missing meals at work, global PSQI, and BMI. A Spearman correlation is an appropriate statistical analysis when assessing the strength of association between two variables and when at least one variable is measured on an ordinal level [72]. Cohen's (1992) standard was used to assess the strength of the correlation coefficient to determine the strength of the relationship, where coefficients between .10 and .29 represent a small association; coefficients between .30 and .49 represent a medium association; and coefficients above .50 represent a large association or relationship [73].

There was a statistically significant association between average hours of sleep between shifts and BMI, $r_{s}=.35, p=.004$. There was a moderate positive relationship between average hours of sleep between shifts and BMI. There was a statistically significant association between sleep quality and BMI, $r_{s}=.25, p=.043$. There was a small positive relationship between sleep quality and BMI. There was a statistically significant association between frequency of meals missed while working and BMI, $r_{s}=.28, p=.024$. There was a small positive relationship between frequency of missing meals while working and BMI. The null hypothesis $\left(H_{0} 1\right)$ for Research Question 1 was partially rejected. Due to the low sample size used in the research, there is a lack of statistical power in the findings [76]. Therefore, the significant and nonsignificant findings interpretation must be with caution. Table 3 presents the findings of the Spearman correlations.

\begin{tabular}{|c|c|c|}
\hline & \multicolumn{2}{|c|}{ BMI } \\
\hline & $\boldsymbol{r}_{\boldsymbol{s}}$ & $\boldsymbol{p}$ \\
\hline Average hours of sleep between shifts & .35 & .004 \\
\hline Sleep quality & .25 & .043 \\
\hline Frequency of missing meals while working & .28 & .024 \\
\hline Global PSQI & .05 & .713 \\
\hline
\end{tabular}

Table 3: Spearman Correlations between Insufficient Sleep, Abnormal Eating Patterns, and BMI

To address research question two, a series of Spearman correlations were conducted to examine the two-way associations between working the night shift, shift extending to 12 hours, length of time in the current shift, and BMI. There was a statistically significant association between length of time working the current shift and BMI, $r_{s}=.30, p=.014$. The sign and strength of the coefficient suggests there was a moderate positive relationship between length of working current shift and BMI. Working the night shift and having shifts extend to 12 hours did not show a significant association on BMI. Due to the significant association between work 
night shift and BMI, the null hypothesis $\left(\mathrm{H}_{0} 2\right)$ for research question two was partially rejected. Due to the low sample size collected for the research, there is a lack of statistical power in the findings [76]. Therefore, the significant and non-significant findings interpretation must be with a level of caution. Table 4 presents the findings of the Spearman correlations.

\begin{tabular}{|c|c|c|}
\hline & \multicolumn{2}{|c|}{ BMI } \\
\hline & $r_{s}$ & $p$ \\
\hline Work night shift & .02 & .855 \\
\hline Shift extend to 12 hours & -.04 & .723 \\
\hline Length of time in current shift & .30 & .014 \\
\hline
\end{tabular}

Table 4: Spearman Correlations between Working Night Shift, Shift Extending to 12 Hours, Length of Time in Current Shift, and BMI

To address research question three, a Spearman correlation was conducted to examine the two-way associations between age and BMI. Age was not statistically associated with BMI level. The findings of the correlation are presented in Table 5.

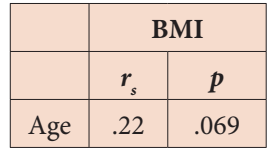

Table 5: Spearman Correlations between Age and BMI

An analysis of variance (ANOVA) was conducted to examine for differences in BMI by marital status. An ANOVA is an appropriate statistical analysis when comparing differences in a continuous dependent variable between groups [77]. The continuous dependent variables in this analysis corresponded to BMI. The independent grouping variables in this analysis corresponded to marital status - single, married, divorced, and widowed.

Prior to analysis, the assumptions of the ANOVA were assessed. Normality of the dependent variables was tested with a Kolmogorov Smirnov (KS) test. The results of the KS test indicated statistical significance for BMI $(p=.006)$; therefore, the assumption was not met for this variable. Although the normality assumption was not met through the KS test, the ANOVA is robust for stringent assumptions when the sample size is sufficiently large $(n>50)$ (Stevens, 2009). Homogeneity of variance was assessed with Levene's test and the results were statistically significant for specialty unit $(p=.611)$; thus, the assumption was met for this variable.

The results of the ANOVA were statistically significant for marital status $\left(F(3,63)=7.09, p<.001\right.$, partial $\left.\eta^{2}=.252\right)$, suggesting that there were statistical differences by marital status. Through examination of post hoc tests via Tukey comparisons, it was determined that married participants had significantly higher BMI levels in comparison to single and divorced participants. Due to significant differences in marital status, the null hypothesis $\left(H_{0} 3\right)$ was partially rejected. Because of the low sample size collected for the research, there is a lack of statistical power in the findings. Therefore, the significant findings must be interpreted with a level of caution. Table 6 presents the findings of the ANOVA. Table 7 presents the descriptive statistics of BMI by marital status.

\begin{tabular}{|c|c|c|c|c|c|}
\hline Source & Hypothesis df & Error $d \boldsymbol{f}$ & $\boldsymbol{F}$ & $\boldsymbol{p}$ & $\boldsymbol{\eta}^{2}$ \\
\hline Marital Status & 3 & 63 & 7.09 & $<.001$ & .252 \\
\hline
\end{tabular}

Table 6: ANOVA for BMI by Marital Status

\begin{tabular}{|c|c|c|c|}
\hline Continuous Variables & $\boldsymbol{n}$ & $\boldsymbol{M}$ & $\boldsymbol{S D}$ \\
\hline Marital Status & & & \\
\hline Single & 11 & 23.13 & 2.81 \\
\hline Married & 50 & 26.01 & 2.51 \\
\hline Divorced & 4 & 21.67 & 1.91 \\
\hline Widower & 2 & 22.87 & 4.14 \\
\hline
\end{tabular}

Table 7: Means and Standard Deviations for BMI by Marital Status

To address research question four, a series of Spearman correlations were conducted to examine the two-way associations between stress, decreased physical activity, and BMI. Perceived stress and physical activity did not have a significant association with BMI. The findings of the Spearman correlations are presented in Table 8.

\begin{tabular}{|c|c|c|}
\hline & \multicolumn{2}{|c|}{ BMI } \\
\hline & $\boldsymbol{r}_{\boldsymbol{s}}$ & $\boldsymbol{p}$ \\
\hline Perceived Stress & -.03 & .832 \\
\hline Physical Activity & -.13 & .289 \\
\hline
\end{tabular}

Table 8: Spearman Correlations between Perceived Stress, Physical Activity, and BMI 
An analysis of variance (ANOVA) was conducted to examine for differences in BMI by specialty unit. The continuous dependent variables in this analysis corresponded to BMI. The independent grouping variables in this analysis corresponded to specialty unit - medical surgical unit, telemetry unit, critical care units, operating room, and labor unit.

Prior to analysis, the assumptions of the ANOVA were assessed. Normality of the dependent variables was tested with a Kolmogorov Smirnov (KS) test. The results of the KS test indicated statistical significance for BMI $(p=.006)$; therefore, the assumption was not met for this variable. Although the normality assumption was not met through the KS test, the ANOVA is robust for stringent assumptions when the sample size is sufficiently large $(n>50)$ (Stevens, 2009). Homogeneity of variance was assessed with Levene's test and the results were statistically significant for specialty unit $(p=.017)$; thus, the assumption was not met for this variable. Therefore, the findings of the ANOVA will be interpreted with caution.

The results of the ANOVA were not statistically significant for specialty unit $\left(F(4,62)=2.33, p=.066\right.$, partial $\left.\eta^{2}=.131\right)$, suggesting that there were not statistical differences by specialty unit. Due to non-significance of the correlations and ANOVA, the null hypothesis $\left(H_{0} 4\right)$ for research question four was not rejected. Because of the low sample size collected for the research, there is a lack of statistical power in the findings [76]. Therefore, the non-significant findings interpretation must be with a level of caution. Table 9 presents the findings of the ANOVA. Table 10 presents the descriptive statistics of BMI by specialty unit.

\begin{tabular}{|c|c|c|c|c|c|}
\hline Source & Hypothesis $\boldsymbol{d} \boldsymbol{f}$ & Error $\boldsymbol{d f}$ & $\boldsymbol{F}$ & $\boldsymbol{p}$ & $\boldsymbol{\eta}^{\mathbf{2}}$ \\
\hline Specialty Unit & 4 & 62 & 2.33 & .066 & .131 \\
\hline
\end{tabular}

\begin{tabular}{|c|c|c|c|}
\hline Continuous Variables & $\boldsymbol{n}$ & $\boldsymbol{M}$ & $\boldsymbol{S D}$ \\
\hline Specialty Unit & & & \\
\hline Medical-Surgical Unit & 29 & 24.37 & 2.50 \\
\hline Telemetry Unit & 8 & 24.89 & 4.66 \\
\hline Critical Care Unit & 24 & 26.54 & 2.20 \\
\hline Operating Room & 2 & 24.80 & 1.13 \\
\hline Labor Unit & 4 & 23.73 & 3.91 \\
\hline
\end{tabular}

Table 10: Means and Standard Deviations for BMI

by Specialty Unit

\section{Multiple Linear Regression}

A multiple linear regression was conducted as an ancillary analysis to examine the predictive relationship between the significant variables and BMI. A multiple linear regression is an appropriate statistical analysis when testing the predictive relationship between a group of predictors and a continuous criterion variable [74]. In this analysis, the predictor variables corresponded to average hours of sleep between shifts, sleep quality, frequency of missing meals at work, length of time in current shift and marital status. The continuous criterion variable corresponded to BMI.

Prior to the regression analysis, the assumptions of normality, homoscedasticity, and absence of multicollinearity were tested. The normality assumption was tested by visual interpretation of a normal P-P plot between the expected cumulative probability and the observed cumulative probability. The raw data closely followed the trend line, thus the assumption of normality was met (see Figure 5). Homoscedasticity was tested by visual inspection of a residuals scatterplot. The assumption of homoscedasticity was met due to there not being a recurring pattern in the data (see Figure 6). The absence of multicollinearity assumption was tested by variance inflation factors (VIFs) and the assumption was met due to the predictors not having VIF values greater than 10 [78].

Results of the overall model of the multiple linear regression were statistically significant, $\left(F(5,61)=6.58, p<.001, R^{2}=.350\right)$, suggesting that collectively there was a significant predictive relationship between the independent variables and BMI level. The $R^{2}$ value indicates that approximately $35.0 \%$ of the variance in BMI level can be explained by the predictor variables. Average hours of sleep between shifts $(t=2.05, p=.045)$, frequency of missing meals while working $(t=2.31, p=.025)$, and marital status $(t=$ $3.95, p<.001)$ were significant positive predictors in the model. Because of the low sample size collected for the research, there is a lack of statistical power in the findings [76]. Therefore, the significant and non-significant findings interpretation must be with a level of caution. The results of the multiple linear regressions are presented in Table 11.

\begin{tabular}{|c|c|c|c|c|c|c|}
\hline Source & $\boldsymbol{B}$ & $\boldsymbol{S E}$ & $\boldsymbol{\beta}$ & $\boldsymbol{t}$ & $\boldsymbol{p}$ & $\boldsymbol{V I F}$ \\
\hline Average hours of sleep between shifts & 0.74 & 0.36 & .25 & 2.05 & .045 & 1.34 \\
\hline Sleep quality & -0.05 & 0.50 & -.01 & -0.10 & .917 & 1.55 \\
\hline Frequency of missing meals while working & 0.91 & 0.39 & .24 & 2.31 & .025 & 1.03 \\
\hline Length of time in current shift & -0.04 & 0.22 & -.02 & -0.19 & .847 & 1.30 \\
\hline Marital Status (reference: single) & 3.08 & 0.78 & .47 & 3.95 & $<.001$ & 1.30 \\
\hline
\end{tabular}

Note: $F(5,61)=6.58, p<.001, R^{2}=.350$

Table 11: Results for Regression with Factors Predicting BMI 


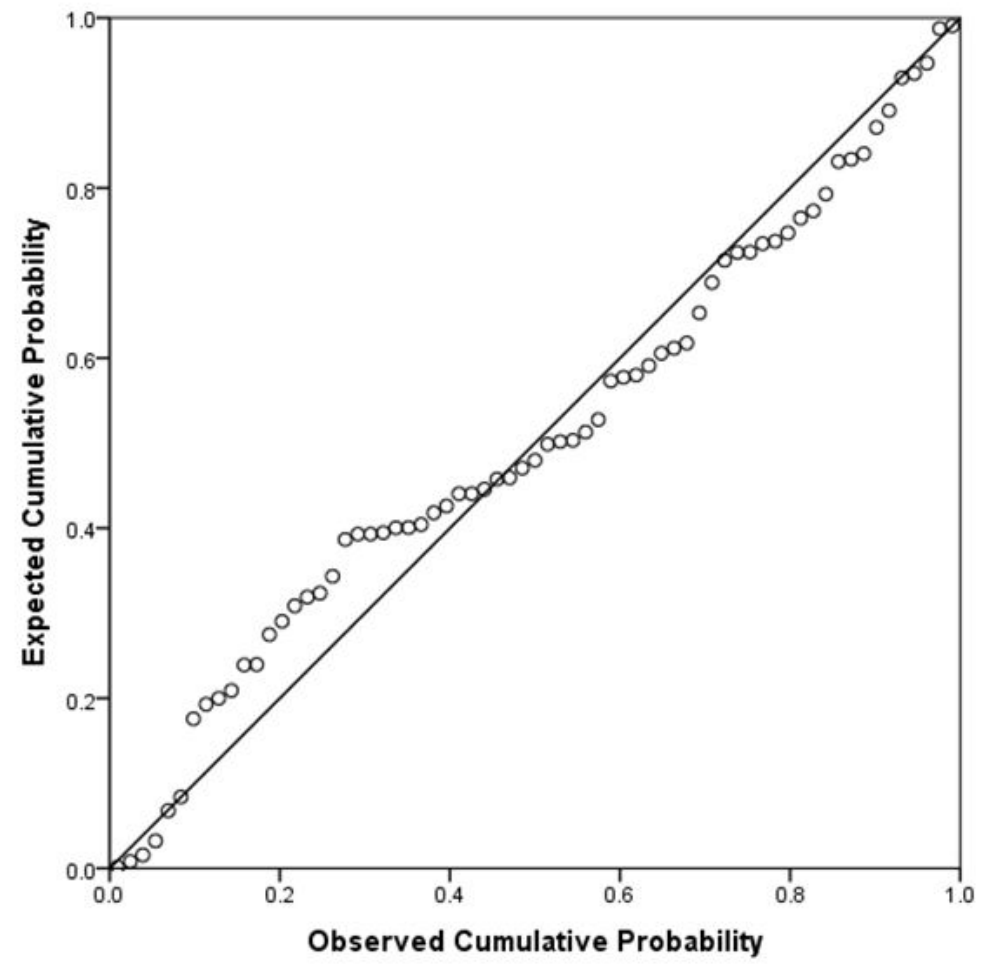

Figure 5: Normal P-P plot for BMI level

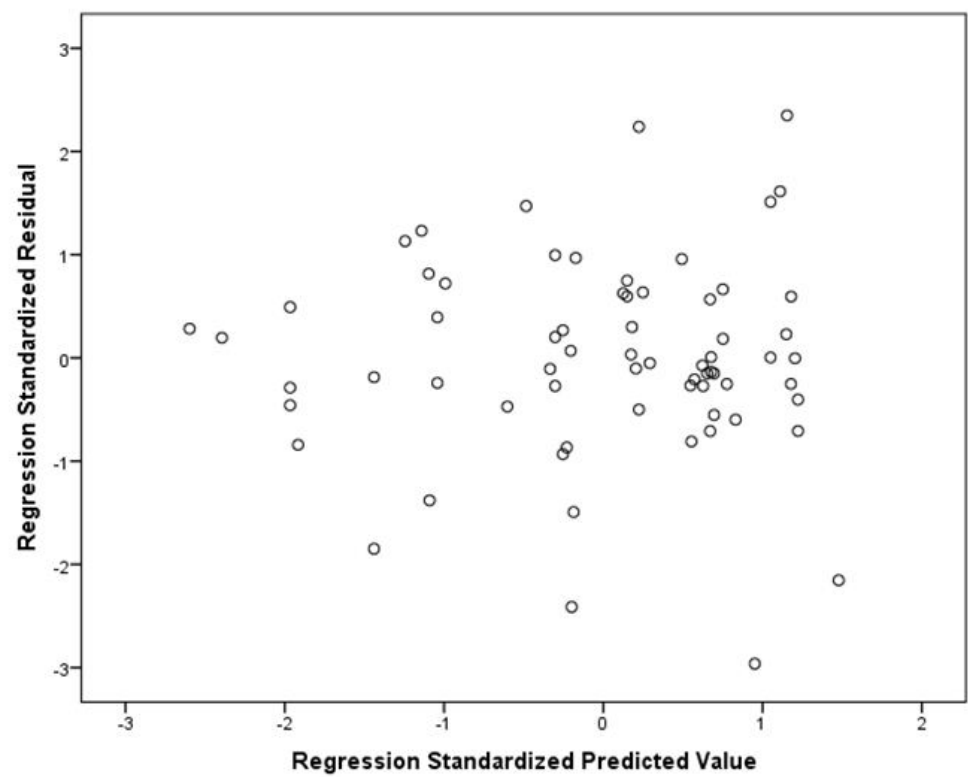

Figure 6: Standardized predicted values versus standardized residuals for the regression on BMI level

\section{Interpretation of the Findings}

I found that there were evidences to partially reject the null hypotheses, which indicated that there were small positive associations between insufficient sleep and abnormal eating patterns with BMI among female Filipino nurses working night shift in the hospital. Because there was a small positive relationship between missed meals and BMI, the null hypothesis was partially rejected.

In this study, I determined that the average hours of sleep between shift were 5 hours $(n=28,42 \%)$ which showed moderate positive relationship with BMI $\left(r_{s}=.35, p=.004\right)$ and also indicated that there was a statistically significant association with BMI $\left(r_{s}=.25\right.$; $p=.043$ ); the most missed meal was dinner $(n=33,49 \%)$, which indicated that there was statistically associations of frequently missed meal with BMI $\left(r_{s}=.28, p=.024\right)$. The findings on insufficient sleep between shifts were confirmed with previous studies from Spaeth et al. (2013), who found that short sleep duration had associations between weight gain and increased in BMI. According to Geiger-Brown et al. (2012), night shift workers had the shortest sleep duration between shifts compared to day shift that increased their risk of weight gain that lead to increase in BMI [13]. The findings on quality of sleep was consistent with the previous studies conducted by Boughattas et al. (2014) and Vijayalaxmi et al. (2014), who reported that $60 \%$ of the night shift 
workers sleep quality were severely compromised and 30\% of them complained of insomnia [3,53]. Also, Kim et al. (2015) found that even a single night of disrupted sleep influenced energy consumption and increased metabolism that promoted frequent eating and increased snacking of high calorie food. The findings on abnormal eating patterns were consistent with previous research from Spaeth et al., (2013), Griep et al., (2014), and Vijayalaxmi et al.,(2014) who found that erratic eating patterns of night shift workers increased energy consumption that promoted increased snacking instead of eating a full meal, which was suggestive of metabolic imbalances that lead to weight gain $[10,38,53]$.

I found that 12-hour shifts and length of time on night shift had significant associations with BMI. However, having worked extended hours beyond 12 hours did not show significant associations on BMI. The null hypothesis, therefore, can be partially rejected due to significant associations between night shift and number of years on night shift with BMI.

In this study, the variables 12-hour shifts and extended years of working the night shift demonstrated statistically significant associations with BMI $\left(r_{s}=.30, p=.014\right)$. I found $(n=63,94 \%)$ that having continued to work 12-hour shifts, and the length of working on night shift showed $(n=20)$ nurses who have worked for 3 to 5 years, $30 \%$, and $(n=16)$ nurses who have worked for 21 to 35 years $(24 \%)$.

In the findings on 12-hour shifts and number of years working the night shift, I found significant associations with BMI, which was confirmed from previous research findings. For example, Kim et al. (2013) found that the longer the nurses worked on night shift, the greater their risk of becoming overweight and obese [8]. Also, Buss (2012) reported that weight gain was higher among night shift nurses [2]. Other researchers have found that extended years of working the night shift caused health problems [34], such as hypertension, diabetes, heart diseases, some forms of cancer, and early disability [12]. In addition, Gu et al. (2015) reported that women who worked night shift for extended period of time had significantly contributed to weight gain and increased BMI [43]. Furthermore, Kim et al. found that long years of working the night shift demonstrated significant associations with increased BMI. Also, Vimalananda et al. (2015) found in African American women who worked the night shift for long durations had significant associations with increased BMI [55].

I found that age of female Filipino nurses showed no statistical association with BMI. However, marital status, particularly married participants, had significantly higher BMI levels compared to single and divorced participants. Due to significant differences in marital status, the null hypotheses were partially rejected.

In this study, I did not find a statistical relationship with age and levels of BMI $\left(r_{s}=.22, p=.069\right)$; however, the results of the ANOVA were significantly associated with $\operatorname{BMI}\left(F(3,63)=7.08, p<.001\right.$. partial $\left.n^{2}=.252\right)$. This study's results on age was not aligned with the previous studies form Letvak et al. (2013), who found that the age of nurses is factor that contributes to higher BMI compared to younger nurses [54]. Contrary to the results of this study on age, Vimalananda et al. (2015) found that women younger than 50-years-old gained weight and became diabetics than those who never work night shift [55]. Likewise, Boughattas et al., (2014) reported that nurse's age influenced sleep quality and duration that were significantly associated with weight gain and changed in BMI [3].

In this study, I found significant associations with marriage and levels of BMI. This result was confirmed form the previous research of Kim et al. (2013), who found in that female married nurses had significant high risk of overweight and obesity [8]. Mata et al. (2015) found that married female had higher BMI than those who never married [56].

I failed to find significant associations between stress, nursing units, and decreased physical activity with BMI among female Filipino nurses working the night shift in the hospital; therefore, the null hypotheses not rejected.

In this study, the analysis on perceived stress $\left(r_{s}=.03, p=.832\right)$, decreased physical activity $\left(r_{s}=.13\right.$, $\left.p=.289\right)$, and nursing units $\left(F(4,62)=2.33, p .066\right.$, partial $\left.n^{2}=.131\right)$ failed to show significant associations with BMI of female Filipino nurses working the night shift in the hospital. These findings were not aligned with previous studies from Buss (2012); Vijayalaxami, George, and Nambiar (2014); Coomarasamy et al. (2014); and Han, Choi-Kwon, and Kim (2015) who found that stress and night shift work were strongly influenced nurses by unhealthy eating habits of high calorie food that caused weight gain. With regards to specialty units, such as ER \& ICU [3]; ICU, ER, and medical/surgical units (Dagget et al., 2016) found that night shift nurses worked on these nursing units were significantly stressed and impacted their unhealthy eating habits of high calorie food that led them to gain weight. Also, Amani and Gill (2013), found in their previous research that stress and night shift work had strong associations with increased BMI [14].

Many studies proved that physical activity has been identified as having tremendous benefits to overall health [24,47]. However, decreased physical activity is one of the many important factors that caused weight gain, overweight, and obesity [24]. The study findings failed to show the correlation of decreased physical activity with BMI among female Filipino nurses working the night shift in the hospital that is contrary to the previous studies. According to Vijayalaxmi et al. (2014) and Coomarasamy et al. (2014), night shift workers suffered physical exhaustion from work that hindered their physical ability to engage in any form of exercise [24,53]. Likewise, Kim et al., (2013) found from previous research that decreased physical activity had strong significant associations with increased BMI among night shift workers [8]. 


\section{Limitations of the Study}

This study has several limitations. First, due to cross-sectional design, the causality between occupational factors, such as night shifts, meals missed, 12-hour shift, quantity and quality of sleep, weight gain, overweight, and obesity cannot be ascertained [31]. Second, this study completely relied on the self-reported data, which may be suggestive of data inaccuracy that weakens the study validity [65]. Social desirability is another factor of bias that leads to under-reporting of weight as a determinant of BMI [65]. Third, this study has a small sample size and participants were conveniently selected. The result may be choice biases [66]. Because of the study sample was drawn from one hospital and the heterogeneity of the participants, the findings limit the generalizability or comparison to different racial population and different cultures. Finally, some possible confounders of overweight and obesity were not part of the study, such as dietary habits, smoking, alcohol use, and caffeine use [54]. These confounders may provide confounding effect related to weight gain and obesity among female Filipino nurses working the night shift in the hospital.

\section{Recommendations}

This appears to be the first study conducted among female Filipino nurses working the night shift in the hospital. Because this study employed a cross-sectional design, longitudinal and cohorts studies are recommended for future study with this population. This study was focused on the night shift on a single population comprised of female nurses. Future cross-sectional study with the day shift and other racial population and different culture is highly recommended to compare the study result. Literature showed that overweight and obesity had been reported in different countries, such as South Africa, Australia, the United Kingdom, and New Zealand [20], Japan [22], the Republic of Korea [23], Malaysia [24], the United States [17], Brazil [18], Republic of Taiwan [6], Europe [25], and in Canada [26].

Based upon the results of this study, insufficient sleep, married nurses, erratic eating patterns, a working 12-hour shift, and extended years of working on night shift were correlated to weight gain associated with BMI among female Filipino nurses. It is recommended that night shift nurses must play an active role in promoting their health in order to prevent the development of chronic medical conditions, such as obesity, diabetes, hypertension, stroke, heart disease, and early disability [12,29]. It is also recommended that hospital administrators and nursing leaders need to consider changing policies on working conditions within the organization. Some of the suggested changes in organizational policies may include allowing nurses frequent breaks to decrease work fatigue and prevent medication errors $[13,44]$; focus on nutrition and dietary education and provide healthier food choices at the hospital cafeteria [60]; provide working schedules that reduce night shift work [21] and encourage nurses to get sleep more than seven hours per day [44]. Finally, nurses should be encouraged to consult with their health care providers on a regular basis for optimal health [12].

\section{Implications for Social Change}

At the individual level, the potential social change as a result of this study is the provision of important information that is critical for all nurses to help understand the negative implications of night shift work on health. Nurses are equipped with knowledge on health promotion and disease prevention [44], but may not be aware of the negative implications of night shift work. Information from this study enables nurses to take care of their health and prevent the development of chronic medical conditions, such as obesity, diabetes, hypertension, heart diseases, and stroke, some forms of cancer, early disability, and premature death (R. Apellido, personal statement, July 17, 2017). Maintaining and improving the health of the nursing workforce has positive impact in their ability to provide quality care to patients [44]. According to Huth et al. (2012), institution-wide education on the importance of quantity and quality of sleep has positive implications on the health of nurses working the night shift [17]. At the organizational level, information from this study could facilitate change in policies and improve working conditions that promote the health and wellbeing of nurses as discussed in the previous section. At the local, state, and federal level, the potential social change as a result of this study is providing the information critical to public education and health promotion in the prevention of overweight and obesity that may help solve problems with overweight and obesity epidemic. Health promotion and disease prevention at these levels help reduce medical expenditures to the current health care system.

\section{Conclusion}

There is a large body of evidence from previous studies that the cause of unhealthy weight gain or overweight and obesity is multifactorial. Nurses are equipped with adequate knowledge on health promotion and disease prevention and they provide education to their patients as well as the general population. However, this study showed that night shift female Filipino nurses are faced with unhealthy weight gain and increased in BMI. Findings from this study proved that occupational factors, such as insufficient sleep between shifts, abnormal eating habits, working 12-hour shifts, and extended years of working night shift had significant associations with weight gain and BMI. Future research in a larger sample size is needed to validate the findings from this study and further exploration of occupational factors associated with weight gain and obesity among nurses must be conducted in the future. Finally, nurses need to proactively take care of their health so that they can provide quality patient care.

\section{References}

1. Ramin C, Devore EE, Wang W, Pierre-Paul J, Wegrzyn LR, et al. (2015) Night shift work at specific age ranges and chronic disease risk factors. Occup Environ Med 72: 100-7. 
2. Buss J (2012) Association between obesity and stress and shift work among nurses. Workplace Health Saf 60: 453-8.

3. Boughattas W, Olfa EM, Chikh RB, Maoua M, Houda H, et al. (2014) Hospital night shift and its effects on the quality of sleep, the quality of life, and vigilance troubles among nurses. Int J Clin Med 5: 572-83.

4. Buja A, Zampieron A, Mastrangelo G, Petean M, Vinelli A, et al. (2013) Strain and health implications of nurses' shift work. Int J Occup Med Environ Health 26: $511-21$.

5. Deori D (2012) The experience of night shift registered nurses in an acute care setting: A phenomenological study. Int J Latest Res Sci Tech 1: D201-4.

6. Lin PC, Chen CH, Pan SM, Pan CH, Chen CJ, et al. (2012) A typical work schedules are associated with poor sleep quality and mental health in Taiwanese female nurses. Int J Occup Med Environ Health 85: 877-84.

7. Patterson PD, Buysse DJ, Weaver MD, Callaway CW, Yealy DM (2015) Recovery between work shifts among emergency medical services clinicians. Prehosp Emerg Care 19: 365-75.

8. Kim TW, Jeong JH, Hong SC (2015) The impact of sleep and circadian disturbance on hormones and metabolism. Int J Endocrinology 10.1155/2015/591729.

9. Depner CM, Stothard ER, Wright KP Jr (2014) Metabolic consequences of sleep and circadian disorders. Curr Diab Rep Rep 14: 507.

10. Spaeth AM, Dinges DF, Goel N (2013) Effects of experimental sleep restriction on weight gain, caloric intake, and meal timing in healthy adults. Sleep 36: 981-90.

11. Hawkes C, Smith TG, Jewell J, Wardle J, Hammond RA, et al. (2015) Smart food policies for obesity prevention. Lancet 385: 2410-21.

12. Centers for Disease Control and Prevention (2015) Adult obesity facts, USA.

13. Geiger-Brown J, Rogers VE, Trinkoff AM, Kane RL, Bausell RB, et al. (2012) Sleep, sleepiness, fatigue, and performance of 12-hour-shift nurses. Chronobiol Int 29: 211-9.

14. Amani R, Gill T (2013) Shift working, nutrition, and obesity: Implications for workforce health- a systematic review. Asia Pac J Clin Nutr $22: 505-15$.

15. Han K, Trinkoff AM, Storr CL, Geiger-Brown J, Johnson KL, et al. (2012) Comparison of job stress and obesity in nurses with favorable and unfavorable work schedule. J Occup Environ Med 54: 928-32.

16. Nahm ES, Warren J, Zhu S, An M, Brown J (2012) Nurses' self-care behaviors related to weight gain and stress. Nurs Outlook 60: E23-31.

17. Huth JJ, Eliades A, Handwork C, Englehart JL, Messenger J (2012) Shift work, quality of sleep, and elevated body mass index in pediatric nurses. J Pediatr Nurs 28: e64-e73

18. Marquezea EC, Lemosa LC, Soaresa N, Lorenzi-Filhob G, Morenoa CR (2012) Weight gain in relation to night work among nurses. Work 41: 2043-8.

19. Theorell-Haglöw J, Berglund L, Janson C, Lindberg E (2013) Sleep duration and central obesity in women- differences between short sleepers and long sleepers. Sleep Med 13: 1079-85.

20. Phiri LP, Draper CE, Lambert EV, Kolbe-Alexander TL (2014) Nurses' lifestyle behaviors, health priorities and barriers to living a healthy lifestyle: A qualitative descriptive study. BMC Nursing 13: 38.

21. Asaoka S, Aritake S, Komada Y, Ozaki A, Odagiri Y, et al. (2013) Factors associated with shift work disorder in nurses working with rapid-rotation schedules in Japan: the nurses' sleep health project. Chronobiol Int 30: 628-36.

22. Nagashima, S, Masutani, E, Wakamura T (2014) Food intake, behavior, and chronotype of Japanese nurses working irregular shifts. Int J Psychol Stud 6: 107-16.

23. Jung HS, Lee B (2015) Contributors to shift work tolerance in South Korean nurses working rotating shift. Appl Nurs Res 28: 150-5.

24. Coomarasamy JD, Wint NN, Neri DL, Sukumanan S, (2014) Prevalence of obesity and daily lifestyles of registered nurses in Malaysia. Int J Innova App Stud 7: $1202-8$.

25. Saksvik-Lehouillier I, Bjorvatn B, Hetland H, Sandal GM, Moen BE, et al. (2012) Individual, situational and lifestyle factors related to shift work tolerance among nurses who are new and experienced on night work. J Adv Nurs 69: 1136-46.

26. Smith P, Fritschi L, Reid A, Mustard C (2012) The relationship between shift work and body mass index among Canadian nurses. Appl Nurs Res 26: 24-31.

27. Lozano-Kühne JP, Aguila MER, Manalang Jr GF, Chua RB, Gabud RS, et al. (2012) Shift work research in the Philippines: current state and future directions. Philippine Science Letters 5: 17-29.

28. Zimberg IZ, Dâmaso A, Del Re M, Carneiro AM, de Sá Souza H, et al. (2012) Short sleep duration and obesity: Mechanisms and future perspectives. Cell Biochem Funct 30: 524-9.

29. World Health Organization (2015) Overweight and obesity, Sweden.

30. Welford C, Murphy K, Dympna C (2012) Demystifying nursing research terminology: Part 2. Nurse Res 19: 29-35.

31. Sedgwick P (2014) Cross-sectional studies: Advantages and disadvantages. BMJ: 10.1136/bmj.g2276.

32. Krumpal I (2013) Determinants of social desirability bias in sensitive surveys: a literature review. Qual Quant 47: $2025-47$.

33. Chung MH, Kuo TB, Hsu N, Chu H, Chou KR (2012) Recovery after three-shift work: Relation to sleep related cardiac neuronal regulation in nurses. Ind Health 50: 24-30.

34. Guo Y, Liu Y, X, Rong Y, He M, Wang Y, et al. (2013) The effects of shift work on sleeping quality, hypertension, and diabetes in retired workers. PLoS One 8: e71107.

35. Huang TT, Drewnosksi A, Kumanyika S, Glass TA (2009) A system-oriented multilevel framework for addressing obesity in the 21st century. Prev Chronic Dis 6: A82.

36. Chatterji M, Green LW, Kumanyika S (2014) L.E.A.D.: A framework for evidence gathering and use for the prevention of obesity and other complex public health problems. Health Educ Behav 41: 85-99.

37. Kumanyika S, Brownson RC, Cheadle A (2012) The L.E.A.D. framework: Using tools from evidence-based public health to address evidence needs for obesity prevention. Prev Chronic Dis 9: E125.

38. Griep RH, Bastos LS, Fonseca Mde J, Silva-Costa A, Portela LF, et al. (2014) Years worked at night and body mass index among registered nurses from eighteen hospitals in Rio de Janeiro, Brazil. BMC Health Serv Res 14: 603.

39. McFadden E, Jones ME, Schoemaker MJ, Ashworth A, Swerdlow AJ (2014) The relationship between obesity and exposure to light at night: Cross-sectional analysis of over 100,000 women in the Breakthrough Generation Study. Am J Epidemiol 180: 245-50.

40. Beccuti G, Pannain S (2014) Sleep and obesity. Curr Opin Clin Nutr Metab Care 14: 402-12. 
41. Perry GS, Patil SP, Presley-Cantrell LR (2013) Raising awareness of sleep as a healthy behavior. Prev Chronic Dis 10 : E133.

42. Markwald RR, Melanson EL, Smith MR, Higgins J, Perreault L, et al. (2013) Impact of insufficient sleep on total daily energy expenditure, food intake, and weight gain. Proc Natl Acad Sci U S A 110: 5695-700.

43. Gu F, Han J, Laden F, Pan A, Caporaso NE, et al. (2015) Total and cause-specific mortality of U.S. nurses working on rotating shifts. Am J Prev Med 48: 241-52.

44. Caruso CC (2014) Negative impact of shift work and long work hours. Rehabil Nurs 39: 16-25.

45. McNeil J, Doucet É, Chaput JP (2013) Inadequate sleep as a contributor to obesity and type 2 diabetes. Can Diabetes 37: 103-8.

46. Fisharaki G, Kazemnejad A, Zayeri F, Rowzati M, Akbari H (2012) Relatioship between shift work and obesity: A retrospective cohort study. Ir J Mil Med 14: 93-7.

47. Pepłońska B, Burdelak W, Krysicka J, Bukowska A, Marcinkiewicz A, et al. (2014) Night shift work and modifiable lifestyle factor. Int J Occup Med Environ Health 27: 693-706.

48. Smith MR, Eastman CI (2012) Shiftwork: Health, performance and safety problems, traditional countermeasures, and innovative management strategies to reduce circadian misalignment. Nat Sci Sleep 4: 111-32.

49. Kim MJ, Son KH, Park HY, Choi DJ, Yon CH, et al. (2013) Association between shift work and obesity among female nurses: Korean Nurses' Survey. BMC Public Health 13: 1204.

50. Quist HG, Christensen U, Christensen KB, Aust B, Borg V, et al. (2013) Psychological work environment factors and weight change: A prospective study among Danish health care workers. BMC Public Health 13: 43.

51. Kwon J, Park JW, Park JS, Kim S, Choi H, et al. (2016) The relationship between night work and involuntary weight gain: Data from the fifth Korean National Health and Nutrition Examination Survey (KNHANES 2010-2012). Ann Occup Environ Med 28: 10.1186/s40557-010-0088-1.

52. Lewis E, Moscar A, King C, Hogan K (2012) Shift work and development of chronic disease. CAND Vital Link 17-22.

53. Vijayalaxmi MK, George A, Nambier N (2014) A study of general health pattern among shiftwork employees in tertiary care hospital. J Acad Indus Res 3: 176-83.

54. Letvak S, Ruhm C, Gupta S (2013) Differences in health, productivity and quality of care in younger and older 'nurses. J Nurs Manag 21: 914-21.

55. Vimalananda VG, Palmer JR, Gerlovin H, Wise LA, Rosenzweig JL, et al. (2015) Night shift work and incident diabetes among African-American women. Diabetologia 58: 699-706.

56. Mata J, Frank R, Hertwig R (2015) Higher body mass index, less exercise, but healthier eating in married adults: Nine representative surveys across Europe. Soc Sci Med 138: 119-27.

57. Gallant AR, Lundgren J, Drapeau V (2012) The night eating syndrome and obesity. Obes Rev 13: 528-36.

58. Giurgiu D, Bardac D (2013) Overweight, obesity, and physical hypercholesterolemia prevalence in medical staff from Romanian academic county hospital: A cross-sectional study. Acta Medica Transilvanica 11: 196-201.

59. Jermendy G, Nádas J, Hegyi I, Vasas I, Hidvégi T (2012) Assessment of cardiometabolic risk among shift workers in Hungary. Health Qual Life Outcomes 10: 18.

60. Imes CC, Burke LE (2014) The obesity epidemic: The USA as a cautionary tale for the rest of the world. Curr Epidemiol Rep 1: 82-8.

61. Cohen S, Kamarck T, Mermelstein R (1983) A global measure of perceived stress. Journal of Health and Social Behavior 24: 385-96.

62. National Institute of Health (2012) How are overweight and obesity diagnosed?, USA.

63. Grove SK, Burns N, Gray JR (2013) The practice of nursing research: Appraisal, synthesis, and generation of evidence (7 $7^{\text {th }}$ Edn) Elsevier, China.

64. Watson R (2015) Quantitative research. Nurs Stand 29: 44-8.

65. Zhang XC, Kuchenke L, Would M, Velten J, Margraf J (2017) Survey methods matters: Online/offline questionnaire and face-to-face or telephone interviews differ. Comput Hum Behav 17: 172-80.

66. Etikan I, Musa S, Alkassim R (2015) Comparison of convenience sampling and purposive sampling. Am J Theo Appl Stat 5: 1-4.

67. Oakes JM (2007) Statistical power \& sample size: some fundamentals for clinician researchers. Essentials of Clinical Research, Springer, USA.

68. Faul F, Erdfelder E, Buchner A, Lang AG (2014) GPower Version 3.1.9 [computer software]. Uiversität Kiel, Germany

69. Hardigan PC, Succar CT, Fleisher JM (2012) An analysis of response rate and economic costs between mail and web-based surveys among practicing dentist: A randomized trial. J Community Health 37: 383-95.

70. Keusch F (2012) How to increase response rates in list-based web survey samples. Soc Sci Com Rev 30: 380-8.

71. Buysse DJ, Reynolds CF 3rd, Monk TH, Berman SR, Kupfer DJ (1989) The Pittsburgh Sleep Quality Index (PSQI): A new instrument for psychiatric research and practice. Psychiatry Res 28: 193-213.

72. Rebekić A, Lončarić Z, Petrović S, Marić S (2015) Pearson’s or spearman's correlation coefficient-which one to use? Poljoprivreda (Osijek) 21 : 47-54.

73. Cohen J (1992) A power primer. Psychol Bull 112: 155-9.

74. Nimon K, Oswald F (2013) Understanding the results of multiple linear regression: beyond standardized regression coefficients. Organizational Research Methods 16: 650-74.

75. Haegele JA, Hodge SR (2015) Quantitative Methodology: A guide for emerging physical education and adapted physical education researchers. Phys Edu 71: 59-75

76. Williams R, Bornmann L (2016) Sampling issues in bibliometric analysis. J Inform 10: 1225-32.

77. Rojewski JW, Lee IH, Gemici S (2012) Use of "t"-Test and ANOVA in Career-Technical Education Research Career and Technical Education Research 37: 263-75.

78. Akinwande MO, Dikko HH, Samson A (2015) Variance inflator factor. As a condition for inclusion of superior variable(s) in regression analysis. Open J Stat 5: 754-67. 


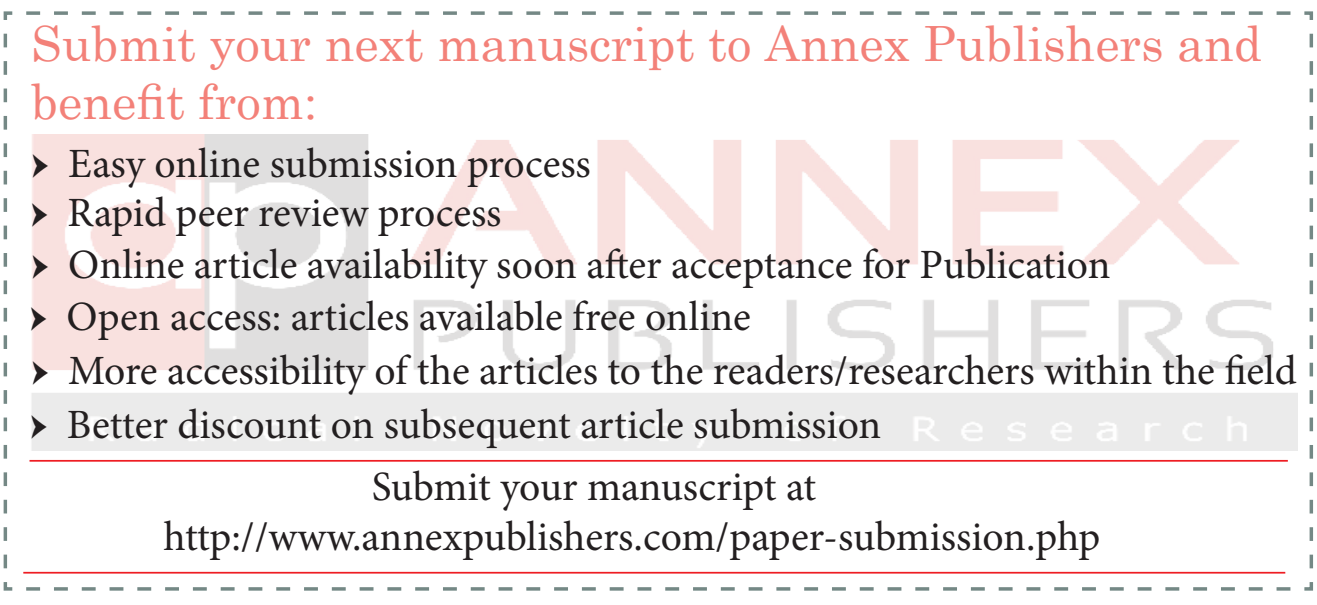

